\title{
Solution of Navier Stokes Equations on a Fixed Mesh using Conforming Enrichment of Velocity and Pressure
}

\author{
D. C. Tanyildiz · J. Marti · R. Rossi
}

Received: date / Accepted: date

\begin{abstract}
Simulation of fluid flows of multi-materials is an intriguing topic in Computational Mechanics. Capturing the physics of the interface between different materials poses a challenge because of the discontinuities that may occur on the interface. Several methods have been proposed in the literature to deal with this issue. In this paper, a technique based on Nitsche's Method has been employed on a fixed mesh combined with the PFEM-2 strategy for the solution of NavierStokes Equations on multi-fluid flows. The novelty of this technique is its capability of capturing the strong and weak discontinuities, and its compatibility for the application of various types of boundary conditions on the interface.
\end{abstract}

Keywords Lagrangian particles · Multi-fluids · Nitsche's Method · Fixed mesh

\section{Introduction}

Multi-fluid flow is a very common phenomena in the nature and therefore its numerical simulation is of great importance. However, this is a challenging task. The first challenge of the simulation of such flows is related with the capturing of the real behaviour of the flow

D. C. Tanyildiz

Centre Internacional de Mètodes Numèrics en Enginyeria (CIMNE)

Gran Capitán s/n, 08034 Barcelona, Spain

E-mail: dcagri@cimne.upc.edu

J. Marti

CIMNE

E-mail: julio.marti@cimne.upc.edu

R. Rossi

CIMNE

E-mail: rrossi@cimne.upc.edu across the interface. This challenge originates from the fact that fluids with different properties may have sharp behaviour changes on the interface. An example for this could be a change in the pressure gradient, or a jump in the velocity field. Apart from the difficulties of capturing these physical changes, another challenge is the difficulty of detecting the evolution of the position of the interface throughout the simulation. To circumvent these issues, special care on the interface has to be taken at each time step.

Several strategies have been developed for the solution of multi-fluid problems. Eulerian based methodologies use special techniques to capture the interface such as: Level Set approaches[1,2], Volume of Fluid methods[3], etc. Since the element could be cut by the interface in any arbitrary position, further techniques, such as X-FEM and E-FEM, are needed for the representation of weak and/or strong discontinuities in the pressure and/or velocity fields $[5,17,20,25,24,26]$. These methodologies exhibit deficiencies in conservation of the mass. Recent studies have introduced novel techniques for alleviating this issue $[6-9,4]$.

A simple remedy to such difficulties is to employ a mesh based Lagrangian approach, and to carry out the simulation by moving the nodes on the interface and changing the mesh topology. [34,36,10-12]. Unfortunately, since the interface is subject to changes, one needs to repeat this procedure in every time-step, leading to a high computational cost. Apart from that, even employing this technique, the nodes at the interface need to be duplicated in order to capture the strong discontinuities across the interface[11]. Moreover, one needs to employ small time steps with mesh based Lagrangian methods in order to avoid the element inversion throughout the simulation. 
Recently in [13-15,28] an embedded approach has been used to model multi-fluid problems. The approach relies on combining an Eulerian and a Lagrangian description for the individual fluids of a multi-fluid problem and solving them in a partitioned manner. Although the methodology exploits the advantages of both formulations, it still faces some limitations. For optimal functionality of the method, the mesh sizes of the involved domains should be similar and the time step size is generally restricted due to the element inversion issue faced in the classical Lagrangian methods.

Following the same idea of the embedded approach, a hybrid Lagrangian-Eulerian variation of the solution strategy called PFEM-2 has been proposed by Idelsohn et al. [18]. While one part of the governing equations are estimated using the particles, the obtained results are projected onto an Eulerian mesh and solved consequently. After that, the estimated Lagrangian solution on the particles is updated according to the results obtained from the mesh. However, an another variation of PFEM-2 which is different than the LagrangianEulerian approach could be found in [29].

The PFEM-2 strategy used in this work has many advantages such as making it possible to use large time steps and therefore having relatively faster solutions and less computational times. Apart from that, the strategy is effective in capturing the interface in multifluid and free-surface flows. However, to deal with the discontinuity issues, a remedy still has to be applied. In [20], an elemental enrichment methodology has been applied to circumvent the issues regarding the discontinuity of the pressure gradient. Although this remedy was successful, it was still not capable of capturing the strong discontinuities in the pressure and/or strong and weak discontinuities in the velocity field. In [24], a new methodology has been proposed to deal with both weak and strong discontinuities in the velocity and the pressure field. However, this methodology was neither applied onto non-stationary problems, nor was employed with the PFEM-2 strategy.

The work which is proposed in this document mainly focuses on a new Nitsche based non-fitting finite element method [21] for circumventing the discontinuity issues in the current PFEM-2 strategy. This methodology has been applied onto the Eulerian part of PFEM-2, and later on, its functionality has been inspected by various test cases. For accomplishing this, the obtained results have been compared with either benchmark solutions or solutions provided by other authors. The structure of this paper is as in the following: Section- 2 provides a brief overview of the governing equations, while Section-3 is dedicated for the explanation of the Second Generation Particle Finite Element Method, the so called PFEM-2. Section-4 explains the issues related to the discontinuous variables and moreover gives insight into the previously mentioned Nitsche based technique [21]. Several test cases and their results are discussed in Section-5. Finally the Section- 6 presents several remarks and the conclusion.

\section{Governing Equations}

Let $\Omega \subset R^{2}$ be a bounded domain containing two fluids in the subdomains $\Omega_{1}$ and $\Omega_{2}$ (Fig.1). The sub-domains are assumed to behave as incompressible viscous fluids. We denote the time by $t$, the Cartesian Spatial Coordinates by $\mathbf{x}=\left.x_{i}\right|_{i=1} ^{2}$, and the vectorial operator of spatial derivatives by $\nabla_{x}=\left\{\partial_{x_{i}}\right\}_{i=1}^{2}$. The evolution of the velocity $\mathbf{v}=\mathbf{v}(\mathbf{x}, t)$ and the pressure $p=p(\mathbf{x}, t)$ is governed by the following equations:

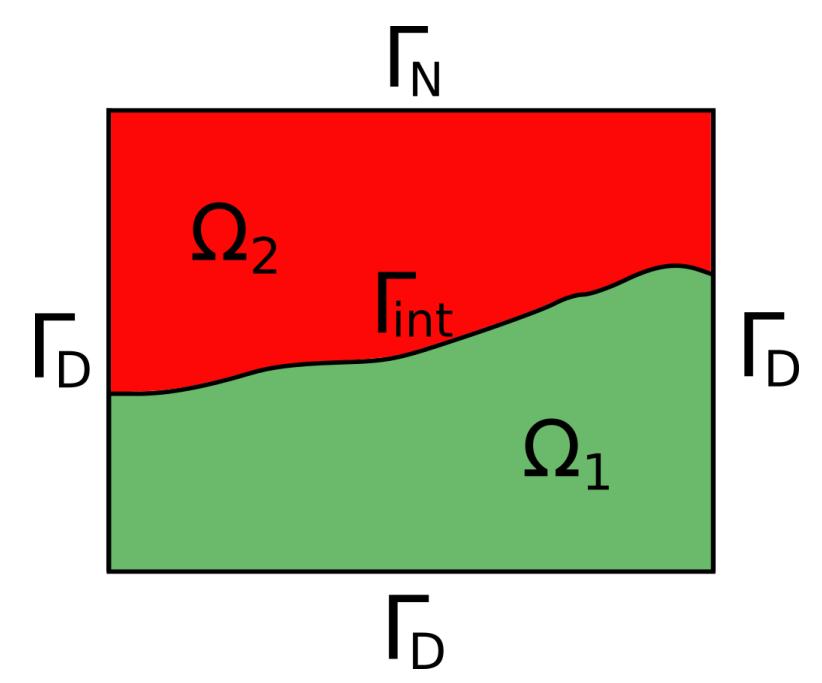

Fig. 1 Bounded domain with two fluids

$\nabla \cdot \mathbf{v}=0$

$\rho \frac{\partial \mathbf{v}}{\partial t}+\rho \mathbf{v} \cdot \nabla \mathbf{v}=\mu \nabla \cdot\left(\nabla \mathbf{v}+\nabla^{T} \mathbf{v}\right)-\nabla p+\mathbf{f}$

where $\mu$ is the dynamic viscosity, $\rho$ is the density, $p$ is the fluid pressure, $\mathbf{v}$ is the velocity and $\mathbf{f}$ is the force, which is calculated by multiplying the body force $\mathbf{b}$ with the density. ( $\mathbf{f}=\rho \mathbf{b})$. Eqs. (1-2) are completed with the boundary conditions, and will be explained more detailed in the sub-section 4.2 . 


\section{First order splitting}

Applying an Euler scheme onto the time differentiation, the following splitting procedure could be carried out:

$$
\frac{\partial \mathbf{v}}{\partial t}=\frac{\mathbf{v}^{n+1}-\mathbf{v}^{n}}{\Delta t}=\frac{\mathbf{v}^{n+1}-\mathbf{v}^{*}+\mathbf{v}^{*}-\mathbf{v}^{n}}{\Delta t}
$$

From now on, to reduce the clutter, the superindex $n+1$ will be neglected. According to this, the Eq. 2 could be rewritten as:

$$
\begin{aligned}
& \rho\left(\frac{\mathbf{v}-\mathbf{v}^{*}}{\Delta t}\right)+\rho\left(\frac{\mathbf{v}^{*}-\mathbf{v}^{n}}{\Delta t}\right) \\
& +\rho \mathbf{v} \cdot \nabla \mathbf{v}-\mu \nabla \cdot\left(\nabla \mathbf{v}+\nabla^{T} \mathbf{v}\right)+\nabla p=\mathbf{f}
\end{aligned}
$$

Assuming that this equation is splittable into two parts, the Eq. 1 and the Eq. 2 could be written as in the following:

$$
\begin{aligned}
& \nabla \cdot \mathbf{v}=0 \\
& \rho\left(\frac{\mathbf{v}-\mathbf{v}^{*}}{\Delta t}\right)-\mu \nabla \cdot\left(\nabla \mathbf{v}+\nabla^{T} \mathbf{v}\right)+\nabla p=\mathbf{f} \\
& \rho\left(\frac{\mathbf{v}^{*}-\mathbf{v}^{n}}{\Delta t}\right)+\rho \mathbf{v} \cdot \nabla \mathbf{v}=0
\end{aligned}
$$

\section{Second Generation Particle Finite Element Method}

This section will introduce the general idea of the PFEM2 strategy. First sub-section will explain the working mechanism of the Lagrangian and the Eulerian part, while the second sub-section will focus on the discrete form of the governing equations on the fixed mesh.

\subsection{Basics of PFEM-2}

PFEM-2 method is a solution strategy for the NavierStokes Equations. It is based on the combination of a Lagrangian step for the solution of the Eq.7, and either an Eulerian or a Lagrangian step for the Eq.5 and the Eq.6. In the context of the work presented in this paper, an Eulerian step has been employed for the solution of the Eq.5 and the Eq.6. These two equations, namely the dynamic Stokes Equations, are solved on a fixed mesh in an Eulerian fashion with an implicit time integration scheme. However, as previously mentioned, this is not the only setting that the strategy could be employed with, rather it is possible to employ the strategy with different configurations. For instance, instead of using a fixed Eulerian mesh, the dynamic Stokes Equations could be solved on a Lagrangian moving mesh as well $[19,29]$. Apart from this, instead of using an explicit method, the time integration of the particles could be carried out using an implicit method, or as well as a mixture of both implicit and explicit methods [19]. Since this is not the main focus of the work presented here, it won't be explained in further detail. However, a detailed information could be obtained through [18].

At the beginning of the simulation, the particles are seeded inside the elements. Each particle represents a material point with properties such as density and viscosity, and carry velocity and position values. Selecting one particle in the whole domain and naming it as $p$ and its position as $\mathbf{x}_{p}$, and furthermore assuming the " $\mathrm{n}$ " is the start and " $\mathrm{n}+1$ " is the end of the time step, calculation of the position of $\mathbf{x}_{p}$ at " $\mathrm{n}+1$ " could be achieved with:

$$
\mathbf{x}_{p}^{n+1}=\mathbf{x}_{p}^{n}+\int_{n}^{n+1} \mathbf{v}^{t}\left(\mathbf{x}_{p}^{t}\right) d t
$$

where $\boldsymbol{x}$ and $\mathbf{v}$ denote the position and the velocity, respectively. A quick inspection of the above equation reveals that, for the calculation of the position, a continuous evaluation of the velocity throughout the timestep is needed. This however is a problem because the velocity which is continuous over time cannot be anticipated a priori. There are various solutions to this problem. In this work, an explicit time integration with streamline integration has been applied to the Eq. 8. In other words, the following has been applied:

$\mathbf{v}^{t}\left(\mathbf{x}_{p}^{t}\right) \approx \mathbf{v}^{n}\left(\mathbf{x}_{p}^{t}\right)$

According to this, the final set of equations would read as:

$\mathbf{x}_{p}^{n+1}=\mathbf{x}_{p}^{n}+\int_{n}^{n+1} \mathbf{v}^{n}\left(\mathbf{x}_{p}^{t}\right) d t$

As previously mentioned, the variation that has been used in this work employs Lagrangian particles for the solution of the convective term, and uses explicit time integration. Since the convective term is solved in a Lagrangian way, explicit schemes are viable without the need to keep the Courant-Lewy Number (CFL) smaller than 1. Therefore, large time-steps could be employed with the strategy so that a fast solution of the NavierStokes simulations could be achieved. However, due to the fact that the integration in the Eq. 10 is not exact ,special care has to be taken regarding the time step size to prevent errors related with the accuracy. Moreover, a quick inspection of the Eq.(6) and the Eq.(7) reveals that neither of these equations has necessarily to be equal to zero, or in other words the splitting introduces an error. This error, which is again related with the accuracy, could be minimized with a correct use of the time step size. As a summary, the choice of the time 
step size in this version of the PFEM-2 strategy is not related with the stability, but with accuracy issues.

The velocities of the particles on the Eq.10 are obtained through the interpolation of the velocity on the mesh, based on their initial positions. Estimating the position of the particles in this explicit way, and projecting the velocity onto the fixed mesh, the Eq.7 is solved through the particles. Subsequently, the governing equations without the convective term, namely the Stokes Equations, Eq.5 and Eq.6, are discretized and solved on the fixed mesh. Later on, as a last step, the velocity values of the particles are updated using the Eulerian Mesh solution. A more detailed explanation on PFEM-2 could be obtained through [37], [19] and $[20]$.

Another important aspect of the strategy is its interface detecting mechanism which operates through particles. As previously explained, the particles carry fluid properties and the velocity. After the projection of the information from the particles onto the mesh, based on the information projected, the interface is detected by looking for the property changes in the element. This is carried out as in the following: Each particle gets a scalar value based on its fluid properties. For instance, if it's air it gets 1, and if it's water it gets the value of -1 . Once the particles are moved, their marker scalar values and as well as the velocities are extrapolated linearly onto the nodes of the elements in a weighted average way using the shape functions. Following the same procedures for all of the particles in the element, the velocities and the scalar values are projected onto the element, and with this scalar value, the position where the interface cuts the element could be detected by searching for the zero values of a pseudo level-set function that is written based on the weighted average extrapolation. More information related to this procedure could be found in [38]. Fig. 2 shows the particles with different material properties and the interface.

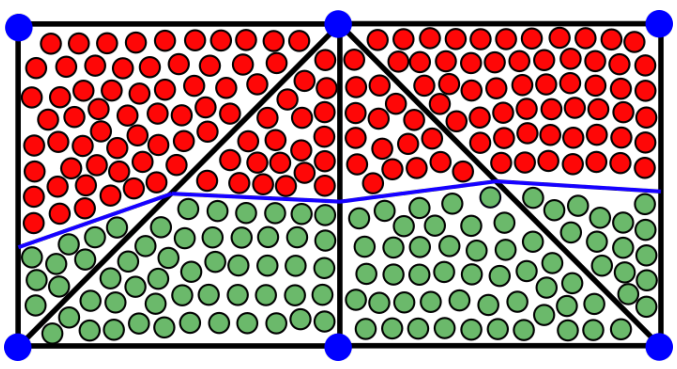

Fig. 2 Particles with different material properties and the interface

The flowchart that summarizes the PFEM-2 strategy has been presented on the Fig. 3. The discretization

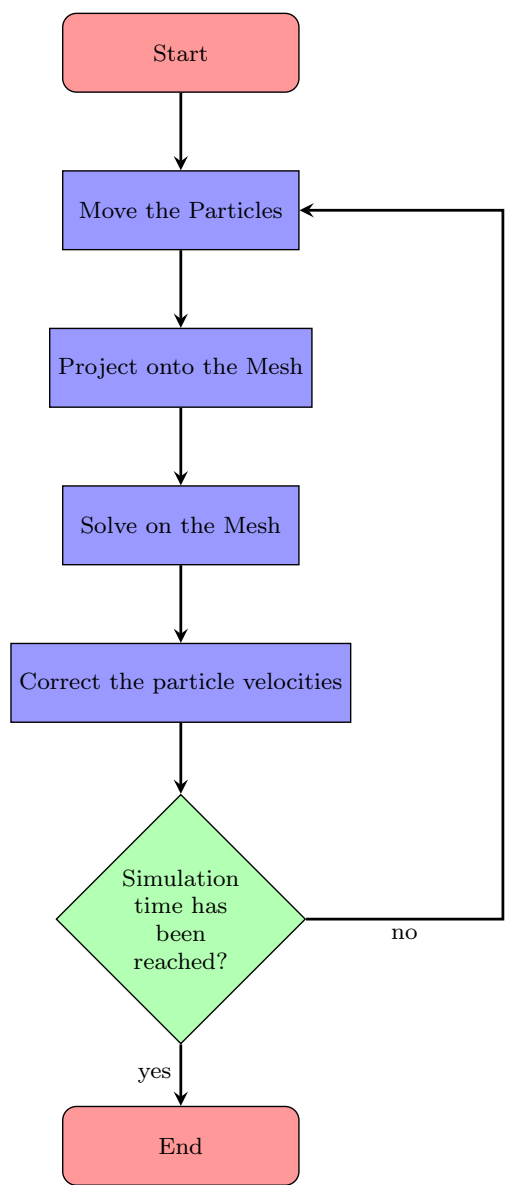

Fig. 3 The flowchart that shows the working mechanism of PFEM-2

of the relevant equations on the Eulerian fixed mesh will be explained in the following sub-section.

\subsection{Discrete Equations on the Fixed Mesh}

Multiplying the Eq. 1 and Eq. 2 with the test functions $q$ and $\boldsymbol{\omega}$ respectively, integrating through the whole domain, and moreover to reduce to clutter using the following Cauchy Stress definition for the Newtonian Fluids:

$\sigma(\mathbf{v}, \mathbf{p})=-p \mathbf{I}+\mu\left(\nabla \mathbf{v}+\nabla^{T} \mathbf{v}\right)$

it is possible to rewrite the equations as in the following:

$$
\begin{aligned}
& \rho\left(\boldsymbol{\omega}, \frac{\partial \mathbf{v}}{\partial t}\right)-(\boldsymbol{\omega}, \nabla \cdot \sigma(\mathbf{v}, \mathbf{p}))=\langle\boldsymbol{\omega}, \mathbf{f}\rangle \\
& (q, \nabla \cdot \mathbf{v})=0
\end{aligned}
$$

Applying integration by parts to the second term of the Eq. 12, the following could be written:

$$
\rho\left(\boldsymbol{\omega}, \frac{\partial \mathbf{v}}{\partial t}\right)+(\nabla \boldsymbol{\omega}, \sigma)-\underbrace{(\boldsymbol{\omega}, \sigma(\mathbf{v}, \mathbf{p}) \cdot \mathbf{n})}_{\text {boundary-term }}=\langle\boldsymbol{\omega}, \mathbf{f}\rangle
$$




$$
(q, \nabla \cdot \mathbf{v})=0
$$

In the notation adopted, $(\cdot, \cdot)$ and $\langle\cdot, \cdot\rangle$ stand for a bilinear and linear forms, respectively. In order to obtain stable solutions, the Eq.15 must be stabilized. This is necessary for equal order velocity-pressure interpolations, as they do not satisfy the compatibility condition [16]. In this work ASGS stabilization technique has been employed [31]. Since the stabilization is out of the scope of this paper, it won't be discussed in further detail.

Considering a finite element linear approximation of the same order for the velocity and the pressure and taking $\mathbf{N}$ as the vector of shape functions, it is possible to write the following:

$p(\mathbf{x})=\mathbf{N}^{T}(\mathbf{x}) \bar{p}$

$\mathrm{v}_{i}(\mathbf{x})=\mathbf{N}^{T}(\mathbf{x}) \overline{\mathrm{v}}_{i}$

Noting that the nodal variables are distinguished from their continuum counterparts by an overbar, the matrices and vectors corresponding to each term of the Galerkin weak form are:

$(\boldsymbol{\omega}, \mathbf{v}) \longrightarrow \mathrm{M} \overline{\mathbf{v}}$

$(\nabla \boldsymbol{\omega}, \nabla \mathbf{v}) \longrightarrow \mathbf{L} \overline{\mathbf{v}}$

$(\nabla \cdot \boldsymbol{\omega}, p) \longrightarrow \mathbf{G} \overline{\mathbf{p}}$,

$\langle\mathbf{f}, \boldsymbol{\omega}\rangle \longrightarrow \mathbf{F}$

Using this notation, the governing equations discretized in space and time using the Backward Euler time integration scheme read:

$$
\left[\begin{array}{cc}
\rho(\mathbf{M} / \Delta t)+\mu \mathbf{L} & \mathbf{G} \\
\mathbf{G}^{T}+\mathbf{S}_{\tau} & \mathbf{L}_{\tau}
\end{array}\right]\left[\begin{array}{l}
\overline{\mathbf{v}} \\
\overline{\mathbf{p}}
\end{array}\right]=\left[\begin{array}{c}
\rho(\mathbf{M} / \Delta t) \mathbf{v}^{n}+\mathbf{f} \\
\mathbf{F}_{\tau}
\end{array}\right]
$$

where $\mathbf{F}_{\tau}, \mathbf{S}_{\tau}$ and $\mathbf{L}_{\tau}$ are the terms related to the stabilization. Further information regarding these terms could be obtained through [30].

The discretized set of equations presented above are not capable of capturing any sort of discontinuity on the interface. The reason for that is the shape functions are continuous over the element, and they are not capable of representing any strong or weak discontinuities. Therefore, a different technique to circumvent this problem is needed. The technique used in this publication and its basis will be explained in the following chapter.

\section{Discontinuous Velocity and Pressure Fields}

Due to the differences in their material properties, the fluids in multi-fluid flows show different physical responses to the ongoing phenomenon, such as weak and strong discontinuities in the unknown field, and specifically in this work, velocity and the pressure.

Here in this publication, a new methodology proposed by [21] is being applied onto the Eulerian part, or in other words the fixed mesh of the domain. Moreover, the necessary continuity and discontinuity on the interface has been enforced using the classical Nitsche's Method [22]. Following subsections will explain this Nitsche based technique in further detail. It should be mentioned that in this work the technique has been implemented only on simplicial 2D triangular elements, with a possible extension to the $3 \mathrm{D}$ tetrahedral elements in the future. The implementation of the methodology on other types of elements could be possible, however has not been studied here.

4.1 Non-fitting Finite Element technique based on Nitsche's Method

This technique is based on dividing the triangular element into two parts, and duplicating the degree of freedoms in each node. The duplicated degree of freedoms will serve as handles to support the continuity of shape functions inside the element. Later on, two sub-local LHS and RHS will be calculated based on the degree of freedoms belonging to each sub-element. Finally, these two sub-local LHS and RHS will be combined into the local ones, and will be merged into the global. A visual explanation is presented on the Fig. 4.

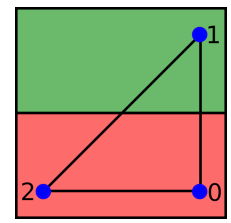

Fig. 4 2D Triangular element with multi-fluids

Fig. 4 represents an interface element taken from a domain where a multi-fluid simulation using PFEM-2 strategy will be conducted. The different colors represent two different fluids with different properties. Nodes are numbered, and it's assumed that at the beginning there is only one degree of freedom, v. However, duplicating this degree of freedom results an additional degree of freedom, which is $\hat{\mathbf{v}}$. Such new degree of freedoms should be considered for all the nodes belonging 
to the elements intersected by the interface. Division of this element according to the method is provided in the Fig. 5.

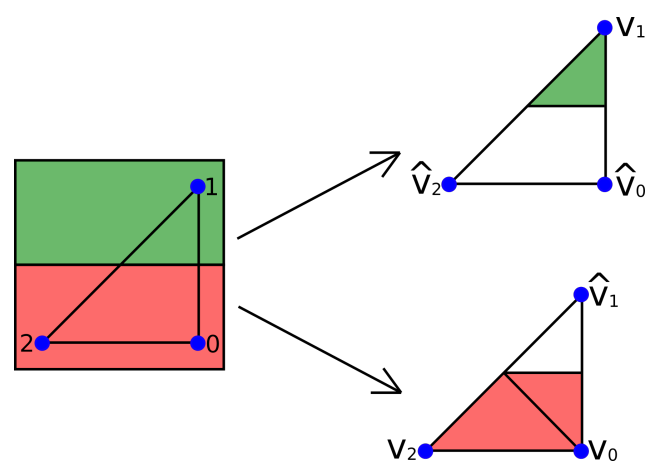

Fig. 5 Division of the element into two sub-elements

It should be noted that, for both of the sub-elements, the integration of the governing equations will be carried out on the part where the fluid pertaining to this sub-element is. In other words, in Fig. 5, the integration will be carried out on the green zone for the sub-element aboe, while for the one below, the equations will be integrated only on the red zone. This mechanism relies on the subdivision of the elements. Element subdivision is however possible only if the interface deos not cross exactly through a node. Therefore to avoid this issue, when the nodes get very close to a node, the distance is pushed with a distance that corresponds to $\% 0.0001$ of the characteristic element size. Fig. 6 is useful for understanding the division of the element into two subelements in macro scale.

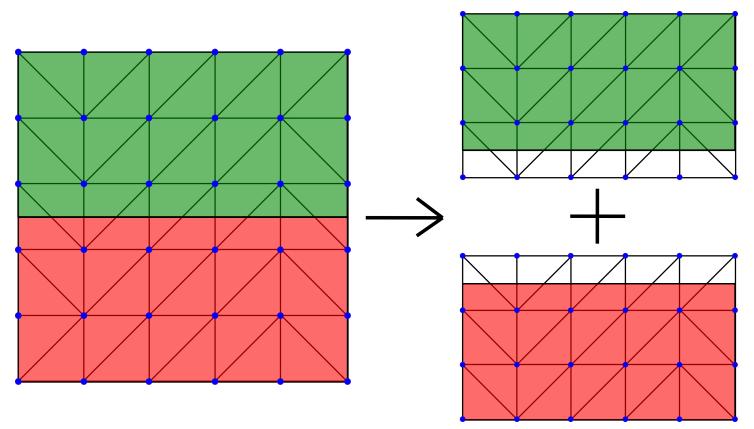

Fig. 6 Division of the whole domain into two sub-domains

The second step, according to the nature of the problem to be solved, is to write a relationship between two interfaces for the two sub-domains. Two subdomains could be completely decoupled from each other, or they could be completely coupled. There are times however that the fluid flow is continuous in the normal direction while being discontinuous in the tangential one, and therefore the sub-domains could be partially coupled. Since there are no extra nodes on the interface, all these particular cases have to be imposed weakly, which is accomplished by the well known Nitsche's Method [22]. Next sub-section will give a brief explanation of the Nitsche's Method and its different application varieties according to the problem to be simulated.

\subsection{Application of Nitsche's method on the interface}

Three types of interface conditions have been applied in this work:

(a) Slip Condition

(b) Continuous Velocity Condition

(c) Continuous Normal Velocity Condition

Note that although more general cases such as the Navier-Slip Boundary Condition [35] could be considered, they were not included in this work.

There are two interfaces in all the cut elements, the interface on the positive and the negative sides. Therefore the conditions mentioned above are applied in both of the interfaces. To reduce the clutter, the explanation will be carried out only on the positive side. However, it should be noted that the same procedure has to be applied on the negative side as well, and eventually the equations for the both sides have to be merged into the local LHS matrix and RHS vector. Apart from this, although the temporal part of the governing equations have been discretized in the preceding section, again to reduce the clutter, it will be used in the continuous form. The Fig. 7 depicts the positive and the negative sides of the domain with two interfaces; and the normal and tangent unit vectors.
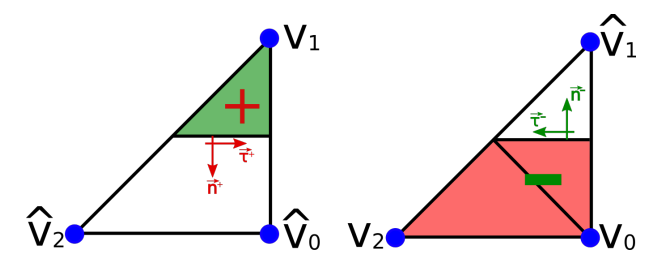

Fig. 7 Sub-elements and the two interfaces on the positive and the negative sides with their outward unit vectors

\subsubsection{Free Slip Condition}

The free-slip condition is the one where the velocity normal to the interface has a constant value, while the component of the traction vector in the direction that is tangent to the interface is zero. In other words, the tangential velocity on the interface is completely free and 
not affected by the interface while the normal velocity is equal to the velocity of the interface in the normal direction. The boundary conditions for this case read as follows:

$\mathbf{v}^{+} \cdot \mathbf{n}^{+}=g$

$\mathbf{t}^{+}(\mathbf{v}, \mathbf{p})-\left(\mathbf{t}^{+}(\mathbf{v}, \mathbf{p}) \cdot \mathbf{n}^{+}\right) \cdot \mathbf{n}^{+}=0$

where $\mathbf{n}^{+}$is the outward unit normal, and the $\mathbf{t}^{+}(\mathbf{v}, \mathbf{p})=\sigma^{+}(\mathbf{v}, \mathbf{p}) \cdot \mathbf{n}^{+}$is the traction vector in the outward direction.

On the positive side of the domain, we would rewrite the Eq. 14 and Eq. 15, where the non-stationary Stokes Equations had been multiplied by the test functions and integrated through the whole domain and moreover integration by parts had been applied onto the Momentum Equation, as in:

$$
\begin{aligned}
& \rho^{+}\left(\boldsymbol{\omega}^{+}, \frac{\partial \mathbf{v}^{+}}{\partial t}\right)_{\Omega_{+}}+\left(\nabla \boldsymbol{\omega}^{+}, \sigma^{+}(\mathbf{v}, \mathbf{p})\right)_{\Omega_{+}} \\
& -\left(\boldsymbol{\omega}^{+}, \sigma^{+}(\mathbf{v}, \mathbf{p}) \cdot \mathbf{n}^{+}\right)_{\Gamma_{+}}=\left\langle\boldsymbol{\omega}^{+}, \mathbf{f}^{+}\right\rangle_{\Omega_{+}} \\
& \left(q^{+}, \nabla \cdot \mathbf{v}^{+}\right)_{\Omega_{+}}=0
\end{aligned}
$$

Separating the boundary term in the Eq. 24 into its components in the normal and the tangential directions, one obtains:

$$
\begin{aligned}
& \rho^{+}\left(\boldsymbol{\omega}^{+}, \frac{\partial \mathbf{v}^{+}}{\partial t}\right)_{\Omega_{+}}+\left(\nabla \boldsymbol{\omega}^{+}, \sigma^{+}(\mathbf{v}, \mathbf{p})\right)_{\Omega_{+}} \\
- & \left(\boldsymbol{\omega}^{+} \cdot \mathbf{n}^{+}, \mathbf{n}^{+} \cdot \sigma^{+}(\mathbf{v}, \mathbf{p}) \cdot \mathbf{n}^{+}\right)_{\Gamma_{+}} \\
- & \left(\boldsymbol{\omega}^{+} \cdot \tau^{+}, \tau^{+} \cdot \sigma^{+}(\mathbf{v}, \mathbf{p}) \cdot \mathbf{n}^{+}\right)_{\Gamma_{+}} \\
= & \left\langle\boldsymbol{\omega}^{+}, \mathbf{f}^{+}\right\rangle_{\Omega_{+}}
\end{aligned}
$$

Applying the condition in the Eq. 23 onto the Eq. 26, the last boundary term cancels out, and the equation further reduces to:

$$
\begin{gathered}
\rho^{+}\left(\boldsymbol{\omega}^{+}, \frac{\partial \mathbf{v}^{+}}{\partial t}\right)_{\Omega_{+}}+\left(\nabla \boldsymbol{\omega}^{+}, \sigma^{+}(\mathbf{v}, \mathbf{p})\right)_{\Omega_{+}} \\
-\left(\boldsymbol{\omega}^{+} \cdot \mathbf{n}^{+}, \mathbf{n}^{+} \cdot \sigma^{+}(\mathbf{v}, \mathbf{p}) \cdot \mathbf{n}^{+}\right)_{\Gamma_{+}}=\left\langle\boldsymbol{\omega}^{+}, \mathbf{f}^{+}\right\rangle_{\Omega_{+}}
\end{gathered}
$$

The Eq. 27 however is not symmetric. To make it symmetric, the symmetric counterpart of the of the boundary term has to be added into the equation. This additional term has to vanish for the correct solution.
To achieve this, it is multiplied with the Dirichlet Boundary Condition in the Eq. 22. Doing this results as in the following:

$$
\begin{aligned}
& \rho^{+}\left(\boldsymbol{\omega}^{+}, \frac{\partial \mathbf{v}^{+}}{\partial t}\right)_{\Omega_{+}}+\left(\nabla \boldsymbol{\omega}^{+}, \sigma^{+}(\mathbf{v}, \mathbf{p})\right)_{\Omega_{+}} \\
- & \left(\boldsymbol{\omega}^{+} \cdot \mathbf{n}^{+}, \mathbf{n}^{+} \cdot \sigma^{+}(\mathbf{v}, \mathbf{p}) \cdot \mathbf{n}^{+}\right)_{\Gamma_{+}} \\
- & \left(\left(\mathbf{v}^{+} \cdot \mathbf{n}^{+}-g\right), \mathbf{n}^{+} \cdot \sigma^{+}(\boldsymbol{\omega}, \mathbf{q}) \cdot \mathbf{n}^{+}\right)_{\Gamma_{+}} \\
= & \left\langle\boldsymbol{\omega}^{+}, \mathbf{f}^{+}\right\rangle_{\Omega_{+}}
\end{aligned}
$$

where for a Newtonian fluid, $\sigma^{+}(\boldsymbol{\omega}, \mathbf{q})=-q^{+} \mathbf{I}+$ $2 \mu^{+} \nabla^{\mathbf{S}} \boldsymbol{\omega}^{+}$.

Adding the penalty term $\alpha\left(\boldsymbol{\omega}^{+} \cdot \mathbf{n}^{+}, \mathbf{v}^{+} \cdot \mathbf{n}^{+}-g\right)$ into the Eq. 28, the application of Nitsche's Method for the Slip Condition is achieved. It should be noted that the penalty term is a sufficiently large term that becomes zero for the correct solution, and it is responsible for ensuring the coercivity in the equations. The final form of the equations for the positive part would read as:

$$
\begin{aligned}
& \rho^{+}\left(\boldsymbol{\omega}^{+}, \frac{\partial \mathbf{v}^{+}}{\partial t}\right)_{\Omega_{+}}+\left(\nabla \boldsymbol{\omega}^{+}, \sigma^{+}(\mathbf{v}, \mathbf{p})\right)_{\Omega_{+}} \\
& -\left(\boldsymbol{\omega}^{+} \cdot \mathbf{n}^{+}, \mathbf{n}^{+} \cdot \sigma^{+}(\mathbf{v}, \mathbf{p}) \cdot \mathbf{n}^{+}\right)_{\Gamma_{+}} \\
& -\left(\left(\mathbf{v}^{+} \cdot \mathbf{n}^{+}-g\right), \mathbf{n}^{+} \cdot \sigma^{+}(\boldsymbol{\omega}, \mathbf{q}) \cdot \mathbf{n}^{+}\right)_{\Gamma_{+}} \\
& +\alpha\left(\boldsymbol{\omega}^{+} \cdot \mathbf{n}^{+},\left(\mathbf{v}^{+} \cdot \mathbf{n}^{+}-g\right)\right)_{\Gamma_{+}}=\left\langle\boldsymbol{\omega}^{+}, \mathbf{f}^{+}\right\rangle_{\Omega_{+}} \\
& \left(q^{+}, \nabla \cdot \mathbf{v}^{+}\right)_{\Omega_{+}}=0
\end{aligned}
$$

Using the same procedure on the negative part of the element (Fig. 7), one obtains:

$$
\begin{gathered}
\rho^{-}\left(\boldsymbol{\omega}^{-}, \frac{\partial \mathbf{v}^{-}}{\partial t}\right)_{\Omega_{-}}+\left(\nabla \boldsymbol{\omega}^{-}, \sigma^{-}(\mathbf{v}, \mathbf{p})\right)_{\Omega_{-}} \\
-\left(\boldsymbol{\omega}^{-} \cdot \mathbf{n}^{-}, \mathbf{n}^{-} \cdot \sigma^{-}(\mathbf{v}, \mathbf{p}) \cdot \mathbf{n}^{-}\right)_{\Gamma_{-}} \\
-\left(\left(\mathbf{v}^{-} \cdot \mathbf{n}^{-}-g\right), \mathbf{n}^{-} \cdot \sigma^{-}(\boldsymbol{\omega}, \mathbf{q}) \cdot \mathbf{n}^{-}\right)_{\Gamma_{-}} \\
+\alpha\left(\boldsymbol{\omega}^{-} \cdot \mathbf{n}^{-},\left(\mathbf{v}^{-} \cdot \mathbf{n}^{-}-g\right)\right)_{\Gamma_{-}}=\left\langle\boldsymbol{\omega}^{-}, \mathbf{f}^{-}\right\rangle_{\Omega_{-}}
\end{gathered}
$$

$\left(q^{-}, \nabla \cdot \mathbf{v}^{-}\right)_{\Omega_{-}}=0$ 


\subsubsection{Continuous Velocity Condition}

A second possible scenario is to require that the velocities in all directions and the traction vector are equal on both of the interfaces. An oil bubble inside the water is a good example for this case. The boundary conditions at the interface for this case are as in the following:

$\mathbf{v}^{+}=\mathbf{v}^{-}$

$\sigma^{+}(\mathbf{v}, \mathbf{p}) \cdot \mathbf{n}^{+}=\sigma^{-}(\mathbf{v}, \mathbf{p}) \cdot \mathbf{n}^{+}$

It should be noted that the Eq. 34 has been written this way by following $\sigma^{+}(\mathbf{v}, \mathbf{p}) \cdot \mathbf{n}^{+}+\sigma^{-}(\mathbf{v}, \mathbf{p}) \cdot \mathbf{n}^{-}=0$ and applying $\mathbf{n}^{-}=-\mathbf{n}^{+}$.

Rewriting the non-stationary Stokes Equations with the boundary terms that appear after integration by parts:

$$
\begin{aligned}
& \rho^{+}\left(\boldsymbol{\omega}^{+}, \frac{\partial \mathbf{v}^{+}}{\partial t}\right)_{\Omega_{+}}+\left(\nabla \boldsymbol{\omega}^{+}, \sigma^{+}(\mathbf{v}, \mathbf{p})\right)_{\Omega_{+}} \\
& -\left(\boldsymbol{\omega}^{+}, \sigma^{+}(\mathbf{v}, \mathbf{p}) \cdot \mathbf{n}^{+}\right)_{\Gamma_{+}}=\left\langle\boldsymbol{\omega}^{+}, \mathbf{f}^{+}\right\rangle_{\Omega_{+}} \\
& \left(q^{+}, \nabla \cdot \mathbf{v}^{+}\right)_{\Omega_{+}}=0
\end{aligned}
$$

And taking the traction equality on the interface, the Eq. 34 into account, the Eq. 35 is modified into the following:

$$
\begin{aligned}
& \rho^{+}\left(\boldsymbol{\omega}^{+}, \frac{\partial \mathbf{v}^{+}}{\partial t}\right)_{\Omega_{+}}+\left(\nabla \boldsymbol{\omega}^{+}, \sigma^{+}(\mathbf{v}, \mathbf{p})\right)_{\Omega_{+}} \\
& -\left(\boldsymbol{\omega}^{+}, \sigma^{-}(\mathbf{v}, \mathbf{p}) \cdot \mathbf{n}^{+}\right)_{\Gamma_{+}}=\left\langle\boldsymbol{\omega}^{+}, \mathbf{f}^{+}\right\rangle_{\Omega_{+}}
\end{aligned}
$$

The next step is to add a symmetric term which vanishes for a correct solution. Adding the symmetric term and multiplying that with the Dirichlet Boundary Condition in the Eq. 33 so that this term vanishes in case of a true solution, following could be written:

$$
\begin{aligned}
& \rho^{+}\left(\boldsymbol{\omega}^{+}, \frac{\partial \mathbf{v}^{+}}{\partial t}\right)_{\Omega_{+}}+\left(\nabla \boldsymbol{\omega}^{+}, \sigma^{+}(\mathbf{v}, \mathbf{p})\right)_{\Omega_{+}} \\
- & \left(\boldsymbol{\omega}^{+}, \sigma^{-}(\mathbf{v}, \mathbf{p}) \cdot \mathbf{n}^{+}\right)_{\Gamma_{+}} \\
- & \left(\left(\mathbf{v}^{+}-\mathbf{v}^{-}\right), \sigma^{-}(\boldsymbol{\omega}, \mathbf{q}) \cdot \mathbf{n}^{+}\right)_{\Gamma_{+}} \\
= & \left\langle\boldsymbol{\omega}^{+}, \mathbf{f}^{+}\right\rangle_{\Omega_{+}}
\end{aligned}
$$$$
\text { where } \sigma^{-}(\boldsymbol{\omega}, \mathbf{q})=-q^{-} \mathbf{I}+2 \mu^{-} \nabla^{\mathbf{S}} \boldsymbol{\omega}^{-} \text {. }
$$

And as the final step, to impose the coercivity in the above equations, adding a sufficiently large penalty term which cancels itself out for the correct solution of the Dirichlet Boundary Condition in the Eq. 33, $\alpha\left(\boldsymbol{\omega}^{+}, \mathbf{v}^{+}-\mathbf{v}^{-}\right)$, non-stationary Stokes Equations with continuous velocity on the interface according to the Nitsche's Method reads as follows:

$$
\begin{aligned}
& \rho^{+}\left(\boldsymbol{\omega}^{+}, \frac{\partial \mathbf{v}^{+}}{\partial t}\right)_{\Omega_{+}}+\left(\nabla \boldsymbol{\omega}^{+}, \sigma^{+}(\mathbf{v}, \mathbf{p})\right)_{\Omega_{+}} \\
& -\left(\boldsymbol{\omega}^{+}, \sigma^{-}(\mathbf{v}, \mathbf{p}) \cdot \mathbf{n}^{+}\right)_{\Gamma_{+}} \\
& -\left(\left(\mathbf{v}^{+}-\mathbf{v}^{-}\right), \sigma^{-}(\boldsymbol{\omega}, \mathbf{q}) \cdot \mathbf{n}^{+}\right)_{\Gamma_{+}} \\
& +\alpha\left(\boldsymbol{\omega}^{+}, \mathbf{v}^{+}-\mathbf{v}^{-}\right)_{\Gamma_{+}}=\left\langle\boldsymbol{\omega}^{+}, \mathbf{f}^{+}\right\rangle_{\Omega_{+}} \\
& \left(q^{+}, \nabla \cdot \mathbf{v}^{+}\right)_{\Omega_{+}}=0
\end{aligned}
$$

Applying the same procedure, the equations for the negative part of the equations read as in the following:

$$
\begin{aligned}
& \rho^{-}\left(\boldsymbol{\omega}^{-}, \frac{\partial \mathbf{v}^{-}}{\partial t}\right)_{\Omega_{-}}+\left(\nabla \boldsymbol{\omega}^{-}, \sigma^{-}(\mathbf{v}, \mathbf{p})\right)_{\Omega_{-}} \\
& -\left(\boldsymbol{\omega}^{-}, \sigma^{+}(\mathbf{v}, \mathbf{p}) \cdot \mathbf{n}^{-}\right)_{\Gamma_{-}} \\
& -\left(\left(\mathbf{v}^{-}-\mathbf{v}^{+}\right), \sigma^{+}(\boldsymbol{\omega}, \mathbf{q}) \cdot \mathbf{n}^{-}\right)_{\Gamma_{-}} \\
& +\alpha\left(\boldsymbol{\omega}^{-}, \mathbf{v}^{-}-\mathbf{v}^{+}\right)_{\Gamma_{-}}=\left\langle\boldsymbol{\omega}^{-}, \mathbf{f}^{-}\right\rangle_{\Omega_{-}} \\
& \left(q^{-}, \nabla \cdot \mathbf{v}^{-}\right)_{\Omega_{-}}=0
\end{aligned}
$$

\subsubsection{Continuous Normal Velocity Condition}

The third condition is the one where the normal velocity is continuous, while the tangential velocity is discontinuous on the interface. Therefore the normal velocities on the interfaces are equal and the normal component of the traction vector on the interfaces sum up to zero. On the other hand, the velocity in the tangential direction is free and the component of the traction vector in the tangential direction is zero. This could be formulated as in the following:

$$
\begin{aligned}
& \mathbf{v}^{+} \cdot \mathbf{n}^{+}=\mathbf{v}^{-} \cdot \mathbf{n}^{-} \\
& \mathbf{t}^{+}(\mathbf{v}, \mathbf{p})-\left(\mathbf{t}^{+}(\mathbf{v}, \mathbf{p}) \cdot \mathbf{n}^{+}\right) \cdot \mathbf{n}^{+}=0 \\
& \mathbf{n}^{+} \cdot \sigma^{+}(\mathbf{v}, \mathbf{p}) \cdot \mathbf{n}^{+}=\mathbf{n}^{+} \cdot \sigma^{-}(\mathbf{v}, \mathbf{p}) \cdot \mathbf{n}^{+}
\end{aligned}
$$

where $\mathbf{n}^{+}$is the outward unit normal, and the $\mathbf{t}^{+}(\mathbf{v}, \mathbf{p})=\sigma^{+}(\mathbf{v}, \mathbf{p}) \cdot \mathbf{n}^{+}$is the traction vector in the outward direction.

Remembering the Eq. 26 where the boundary term was separated into tangent and normal directions: 


$$
\begin{aligned}
& \rho^{+}\left(\boldsymbol{\omega}^{+}, \frac{\partial \mathbf{v}^{+}}{\partial t}\right)_{\Omega_{+}}+\left(\nabla \boldsymbol{\omega}^{+}, \sigma^{+}(\mathbf{v}, \mathbf{p})\right)_{\Omega_{+}} \\
- & \left(\boldsymbol{\omega}^{+} \cdot \mathbf{n}^{+}, \mathbf{n}^{+} \cdot \sigma^{+}(\mathbf{v}, \mathbf{p}) \cdot \mathbf{n}^{+}\right)_{\Gamma_{+}} \\
- & \left(\boldsymbol{\omega}^{+} \cdot \tau^{+}, \tau^{+} \cdot \sigma^{+}(\mathbf{v}, \mathbf{p}) \cdot \mathbf{n}^{+}\right)_{\Gamma_{+}} \\
= & \left\langle\boldsymbol{\omega}^{+}, \mathbf{f}^{+}\right\rangle_{\Omega_{+}}
\end{aligned}
$$

Considering the fact that $\tau^{+} \cdot \sigma^{+}(\mathbf{v}, \mathbf{p}) \cdot \mathbf{n}^{+}$is equal to $\mathbf{t}^{+}(\mathbf{v}, \mathbf{p})-\left(\mathbf{t}^{+}(\mathbf{v}, \mathbf{p}) \cdot \mathbf{n}^{+}\right) \cdot \mathbf{n}^{+}$, and applying the boundary conditions in the Eq. 44 and the Eq. 45, the Eq. 46 turns into:

$$
\begin{aligned}
& \rho^{+}\left(\boldsymbol{\omega}^{+}, \frac{\partial \mathbf{v}^{+}}{\partial t}\right)_{\Omega_{+}}+\left(\nabla \boldsymbol{\omega}^{+}, \sigma^{+}(\mathbf{v}, \mathbf{p})\right)_{\Omega_{+}} \\
- & \left(\boldsymbol{\omega}^{+} \cdot \mathbf{n}^{+}, \mathbf{n}^{+} \cdot \sigma^{-}(\mathbf{v}, \mathbf{p}) \cdot \mathbf{n}^{+}\right)_{\Gamma_{+}} \\
= & \left\langle\boldsymbol{\omega}^{+}, \mathbf{f}^{+}\right\rangle_{\Omega_{+}}
\end{aligned}
$$

Similar to the procedure in the slip condition and the continuous velocity condition, adding a symmetric term multiplied by the dirichlet boundary condition given in the Eq. 43 into the Eq. 47, following is achieved:

$$
\begin{aligned}
& \rho^{+}\left(\boldsymbol{\omega}^{+}, \frac{\partial \mathbf{v}^{+}}{\partial t}\right)_{\Omega_{+}}+\left(\nabla \boldsymbol{\omega}^{+}, \sigma^{+}(\mathbf{v}, \mathbf{p})\right)_{\Omega_{+}} \\
- & \left(\boldsymbol{\omega}^{+} \cdot \mathbf{n}^{+}, \mathbf{n}^{+} \cdot \sigma^{-}(\mathbf{v}, \mathbf{p}) \cdot \mathbf{n}^{+}\right)_{\Gamma_{+}} \\
- & \left(\left(\mathbf{v}^{+} \cdot \mathbf{n}^{+}-\mathbf{v}^{-} \cdot \mathbf{n}^{-}\right), \mathbf{n}^{+} \cdot \sigma^{-}(\boldsymbol{\omega}, \mathbf{q}) \cdot \mathbf{n}^{+}\right)_{\Gamma_{+}} \\
= & \left\langle\boldsymbol{\omega}^{+}, \mathbf{f}^{+}\right\rangle_{\Omega_{+}}
\end{aligned}
$$

And applying the penalty term to enforce the coercivity, $\alpha\left(\boldsymbol{\omega}^{+} \cdot \mathbf{n}^{+}, \mathbf{v}^{+} \cdot \mathbf{n}^{+}-\mathbf{v}^{-} \cdot \mathbf{n}^{-}\right)$, final set of equations could be presented as in the following:

$$
\begin{aligned}
& \rho^{+}\left(\boldsymbol{\omega}^{+}, \frac{\partial \mathbf{v}^{+}}{\partial t}\right)_{\Omega_{+}}+\left(\nabla \boldsymbol{\omega}^{+}, \sigma^{+}(\mathbf{v}, \mathbf{p})\right)_{\Omega_{+}} \\
- & \left(\boldsymbol{\omega}^{+} \cdot \mathbf{n}^{+}, \mathbf{n}^{+} \cdot \sigma^{-}(\mathbf{v}, \mathbf{p}) \cdot \mathbf{n}^{+}\right)_{\Gamma_{+}} \\
- & \left(\left(\mathbf{v}^{+} \cdot \mathbf{n}^{+}-\mathbf{v}^{-} \cdot \mathbf{n}^{-}\right), \mathbf{n}^{+} \cdot \sigma^{-}(\boldsymbol{\omega}, \mathbf{q}) \cdot \mathbf{n}^{+}\right)_{\Gamma_{+}} \\
+ & \alpha\left(\boldsymbol{\omega}^{+} \cdot \mathbf{n}^{+},\left(\mathbf{v}^{+} \cdot \mathbf{n}^{+}-\mathbf{v}^{-} \cdot \mathbf{n}^{-}\right)\right)_{\Gamma_{+}}=\left\langle\boldsymbol{\omega}^{+}, \mathbf{f}^{+}\right\rangle_{\Omega_{+}}
\end{aligned}
$$

$$
\left(q^{+}, \nabla \cdot \mathbf{v}^{+}\right)_{\Omega_{+}}=0
$$

writing the equations for the negative side:

$$
\begin{aligned}
& \rho^{-}\left(\boldsymbol{\omega}^{-}, \frac{\partial \mathbf{v}^{-}}{\partial t}\right)_{\Omega_{-}}+\left(\nabla \boldsymbol{\omega}^{-}, \sigma^{-}(\mathbf{v}, \mathbf{p})\right)_{\Omega_{-}} \\
- & \left(\boldsymbol{\omega}^{-} \cdot \mathbf{n}^{-}, \mathbf{n}^{-} \cdot \sigma^{+}(\mathbf{v}, \mathbf{p}) \cdot \mathbf{n}^{-}\right)_{\Gamma_{-}} \\
- & \left(\left(\mathbf{v}^{-} \cdot \mathbf{n}^{-}-\mathbf{v}^{+} \cdot \mathbf{n}^{+}\right), \mathbf{n}^{-} \cdot \sigma^{+}(\boldsymbol{\omega}, \mathbf{q}) \cdot \mathbf{n}^{-}\right)_{\Gamma_{-}} \\
+ & \alpha\left(\boldsymbol{\omega}^{-} \cdot \mathbf{n}^{-},\left(\mathbf{v}^{-} \cdot \mathbf{n}^{-}+\mathbf{v}^{+} \cdot \mathbf{n}^{+}\right)\right)_{\Gamma_{-}}=\left\langle\boldsymbol{\omega}^{-}, \mathbf{f}^{-}\right\rangle_{\Omega_{-}}
\end{aligned}
$$

$\left(q^{-}, \nabla \cdot \mathbf{v}^{-}\right)_{\Omega_{-}}=0$

\section{Numerical Tests}

This section will provide four numerical tests where different variations of Nitsche's method are applied with the non-fitting Finite Element Method mentioned above. The previously explained Nitsche based non-fitting FEM technique has been implemented onto the Kratos MultiPhysics code, which is an academic Open Source software $[32,33]$. Numerical results will either be compared with other numerical results where a consistent mesh combined with conventional FEM methods are applied, or with cases from bibliography.

Four test cases are presented in the following four sub-sections.

\subsection{Static Elbow Case}

In this numerical example, a static simulation has been conducted using two identical fluids in an elbow shaped domain. The case had previously been studied in [24]. Since the original problem has two different domains with slip-condition on the interface (velocity normal to the wall is zero), to simulate the same physics, the slip condition presented in the sub-section. 4.2.1 has been employed by taking the wall velocity $g$ as $g=0.0$. It should be noted that this is not a Navier-Stokes, but rather a steady Stokes problem. Therefore, no particles have been used, and the solution has only been carried on the fixed mesh. Both fluids have a constant inlet velocity only in the $\mathbf{x}$-direction as $\mathbf{v}_{\mathbf{x}}=1.0 \mathrm{~m} / \mathrm{s}$, and zero velocity in the $\mathbf{y}$-direction as $\mathbf{v}_{\mathbf{y}}=0.0 \mathrm{~m} / \mathrm{s}$. Apart from that, the pressure in the outlet has been fixed to be $p=0.0 P a$, and the velocity in the $\mathrm{x}$-direction to be $\mathbf{v}_{\mathbf{x}}=0.0 \mathrm{~m} / \mathrm{s}$. The domain of the test case is presented on the Fig. 9. The red line in the figure denotes the interface between the two in-miscible fluids, where slip-condition was enforced weakly through Nitsche's Method combined with the methodology discussed in 
the Sec. 4.1, and the normal component of the velocity was set to be $\mathbf{v}_{\mathbf{n}}=0.0$. The cross-sections denoted with the green color, namely the Sec-1 and Sec-2 are the probe points used for the examination of the solution. Sec-1 has been positioned on the cross-section where $x=1.8 m$, while the Sec-2 on $y=5 m$. Both fluids inside the domain have been set to have unit value of dynamic-viscosities and densities, so to say $\rho=1.0 \mathrm{~kg} / \mathrm{m}^{3}$ and $\mu=1.0 \mathrm{~kg} /(\mathrm{ms})$. The methodology has been tested on four different meshes that had the characteristic mesh size as $h=0.01 \mathrm{~m}, h=0.025 \mathrm{~m}$, $h=0.5 \mathrm{~m}$ and $h=0.1 \mathrm{~m}$. The benchmark case, where two seperate domains with a slip boundary condition strongly applied on the interface have been created to solve the problem with conventional FEM, had a characteristic element size of $h=0.01 \mathrm{~m}$. For all of the test cases, an unstructured mesh has been employed. Fig. 10 provides a comparison between the boddy-fitted benchmark mesh vs. the $h=0.1 m$ by zooming in on the bottom right corner of the domain.

Fig. 11 and Fig. 12 show a comparison of the absolutevelocity and the pressure fields between the benchmark solution and the technique that has been used in this research work on the $h=0.025 \mathrm{~m}$ mesh setting. Moreover, the Fig. 13 and Fig. 15 show a comparison of the absolute velocities of the four mesh settings with the benchmark solution, on the section- 1 and section- 2 respectively. Fig. 18 does the same for the pressure field on the Section-2. To make the results more understandable the Fig. 14, Fig. 16, Fig. 17, Fig. 19 and Fig. 20 provide detailed views of the velocity and pressure comparisons. Besides that, a convergence study has been conducted by calculating the error of the methodology solution by comparing the results with the benchmark solution. The error has been calculated by the L2 norm which reads as in the following:

error $=\sqrt{\int\left|\mathbf{v}_{\text {numerical }}-\mathbf{v}_{\text {benchmark }}\right|^{2} d A}$

To estimate the convergence of the methodology, the error has been calculated with four more mesh settings. These mesh settings have been added to have a better understanding of the convergence in the finemesh regime. The additional five mesh settings are: $h=0.02 m, h=0.015 m, h=0.014 m$ and $h=0.011$. The plot is presented in the Fig. 8 .

A quick inspection of the results reveals that the results obtained from the methodology are in good agreement with the benchmark values. Moreover, the Fig. 13, Fig. 15 and Fig. 18 prove that the convergence is achieved through mesh refinement. Finally, an inspection of the

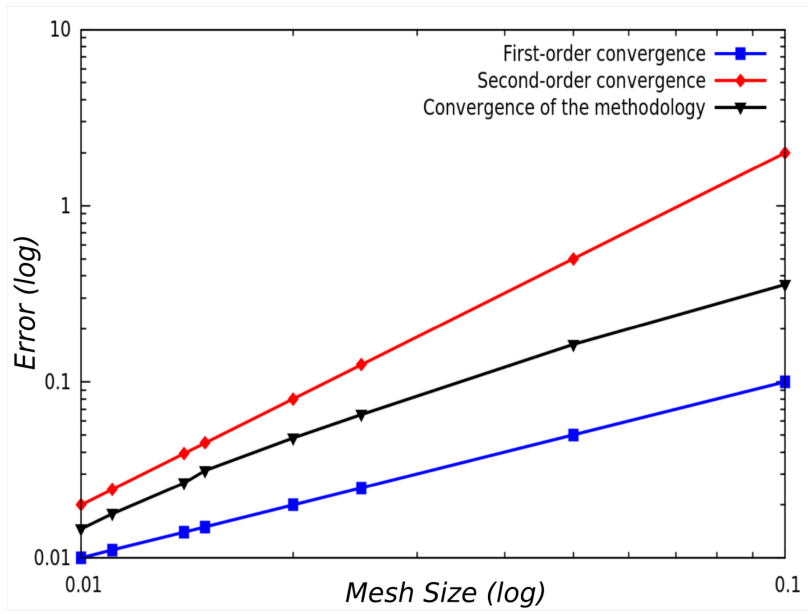

Fig. 8 Convergence study

Fig. 8 reveals that the methodology has a second-order rate of convergence in the fine-mesh region.

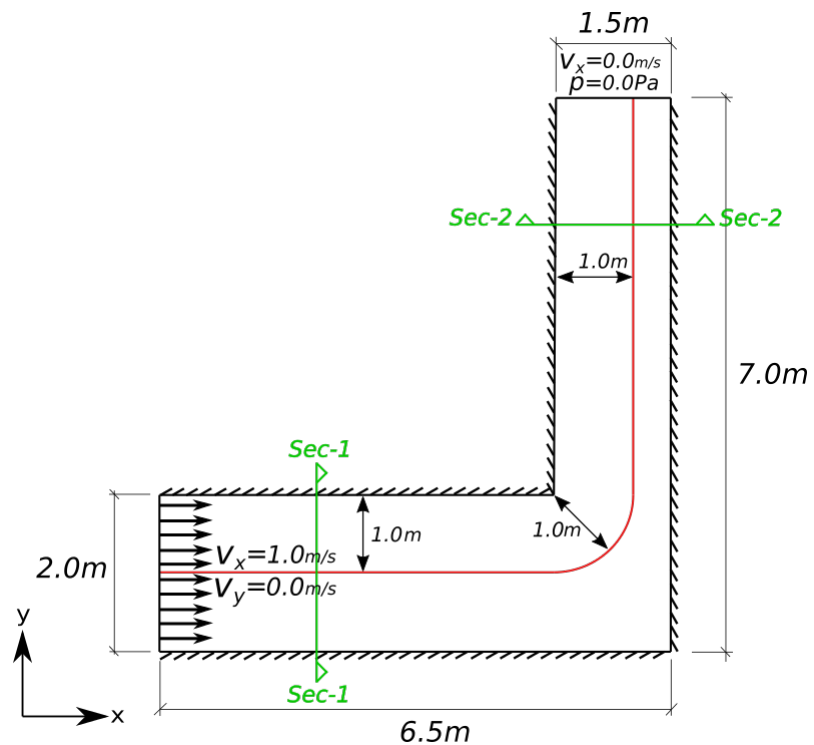

Fig. 9 Elbow domain

\subsection{Taylor-Rayleigh Instability}

Proving the capabilities of the methodology in the static problems, to test it on a full Navier-Stokes simulation, a classical Taylor-Rayleigh Stability case has been employed. For this test case, the results in [23], where a Taylor-Rayleigh Instability problem had been simulated using an Arbitrary Lagrangian-Eulerian formulation, have been used as benchmark. Since the velocity is continuous in both of the domains, the continuous 

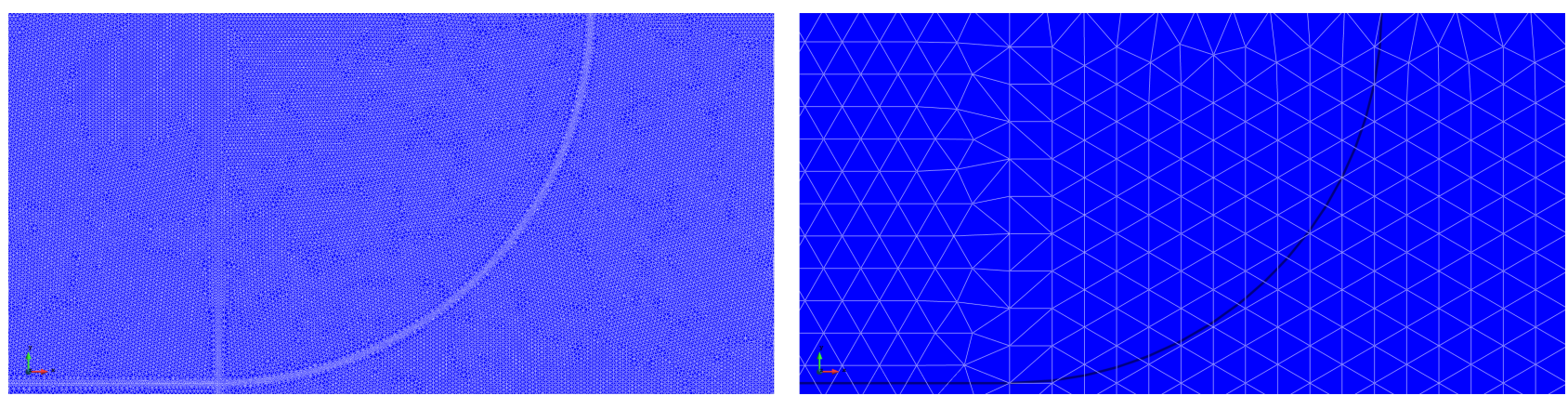

Fig. 10 Benchmark body-fitted mesh on the left and the $\mathrm{h}=0.1 \mathrm{~m}$ mesh setting showing the position of the interface on the right

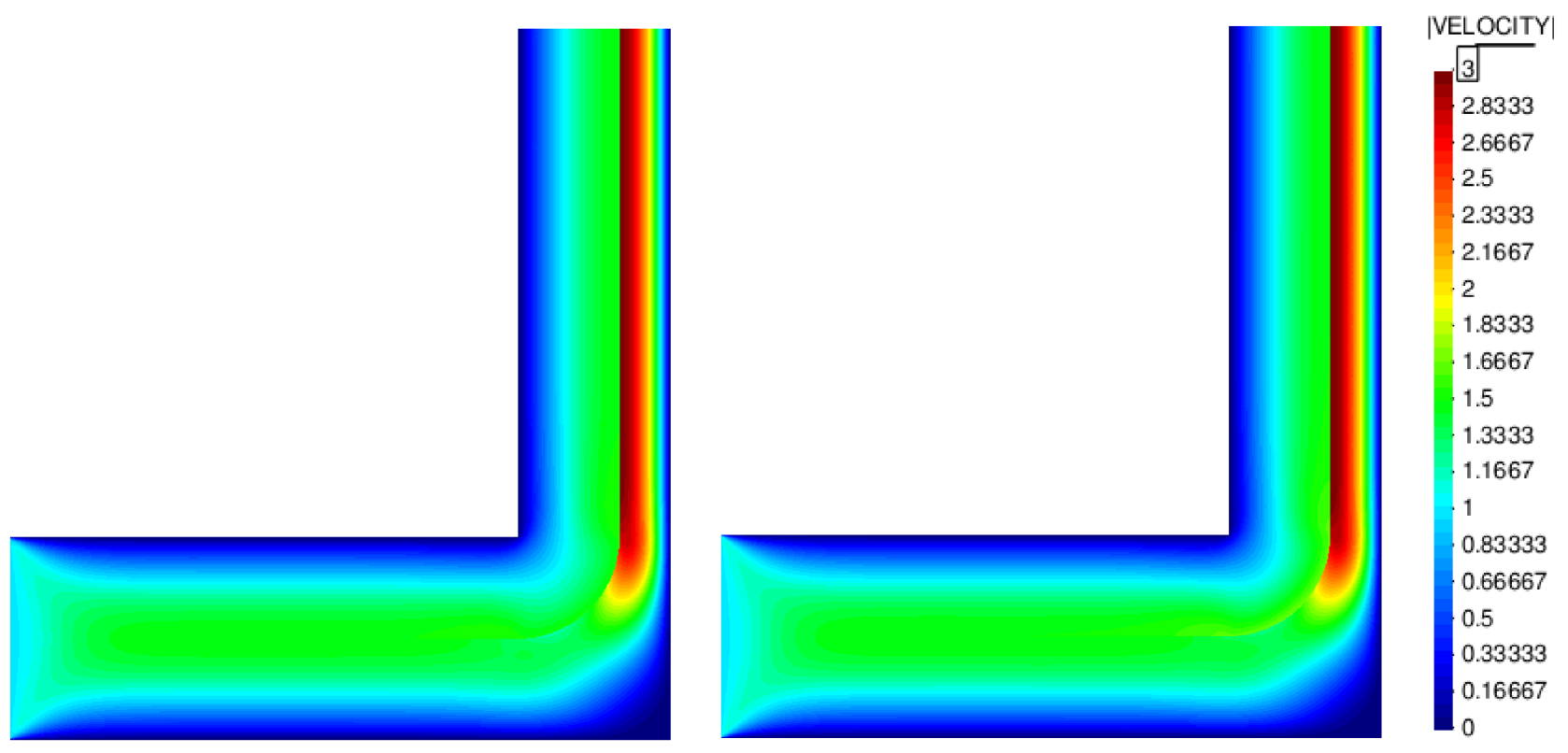

Fig. 11 Absolute velocities of non-fitting technique with the mesh $\mathrm{h}=0.025 \mathrm{~m}$ on the left vs. benchmark on the right
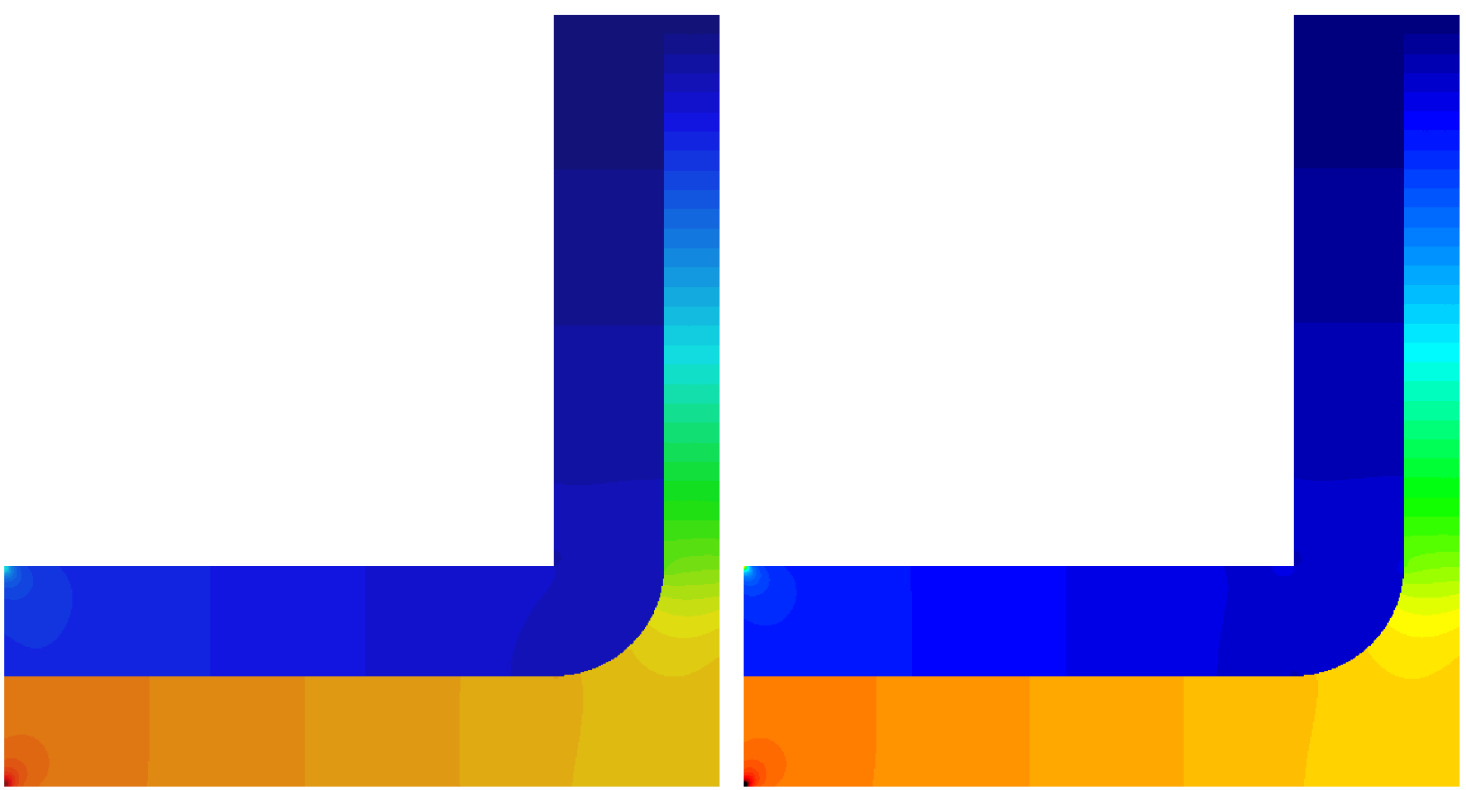

PRESSURE

\begin{tabular}{l}
\hline 210 \\
198.33 \\
186.67 \\
175 \\
163.33 \\
151.67 \\
140 \\
128.33 \\
116.67 \\
105 \\
93.333 \\
81.667 \\
70 \\
58.333 \\
46.667 \\
35 \\
23.333 \\
11.667 \\
0
\end{tabular}

Fig. 12 Pressures of non-fitting technique with the mesh $h=0.025 \mathrm{~m}$ on the left vs. benchmark on the right 


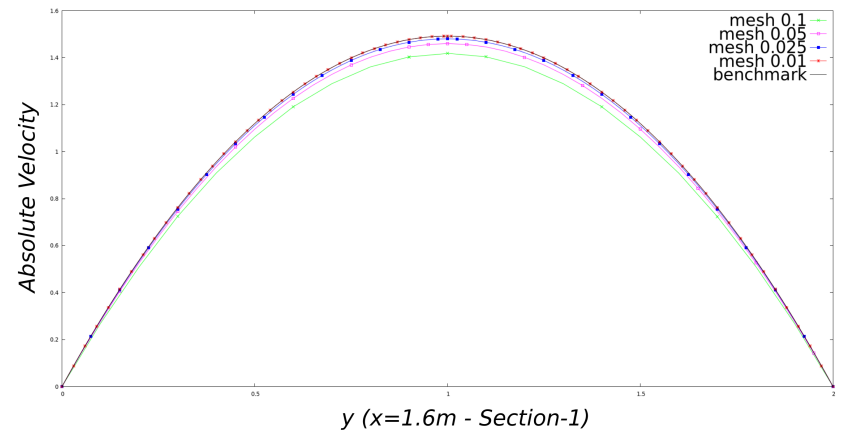

Fig. 13 Comparison of the absolute velocities for different mesh settings and the benchmark solution on the Section-1

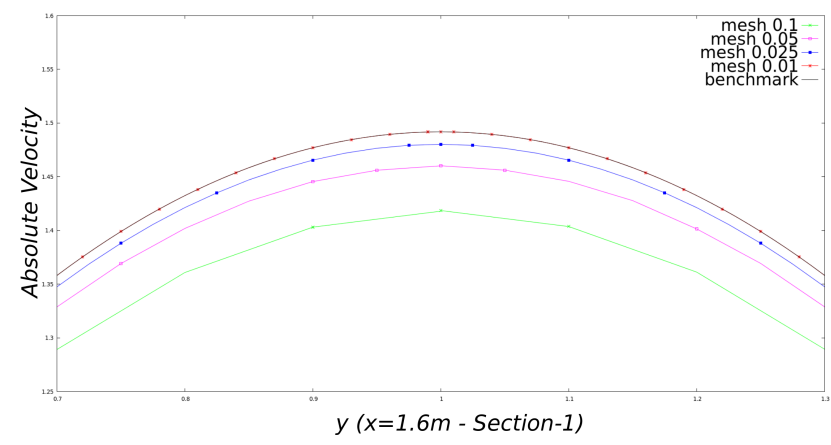

Fig. 14 Detailed view of the Fig. 13

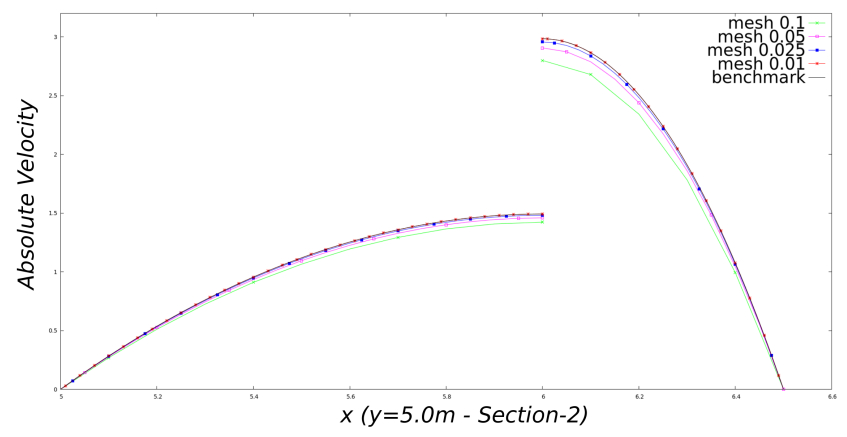

Fig. 15 Comparison of the absolute velocities for different mesh settings and the benchmark solution on the section-2

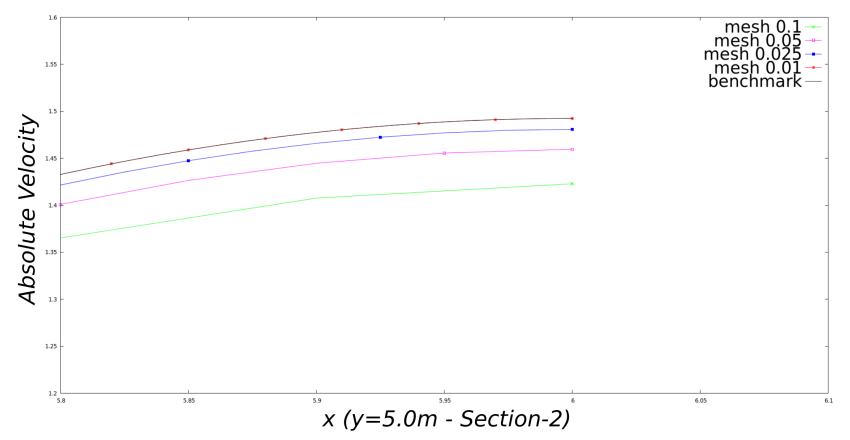

Fig. 16 Detailed view of the Fig. 15

velocity condition of the sub-section. 4.2.2 has been employed.

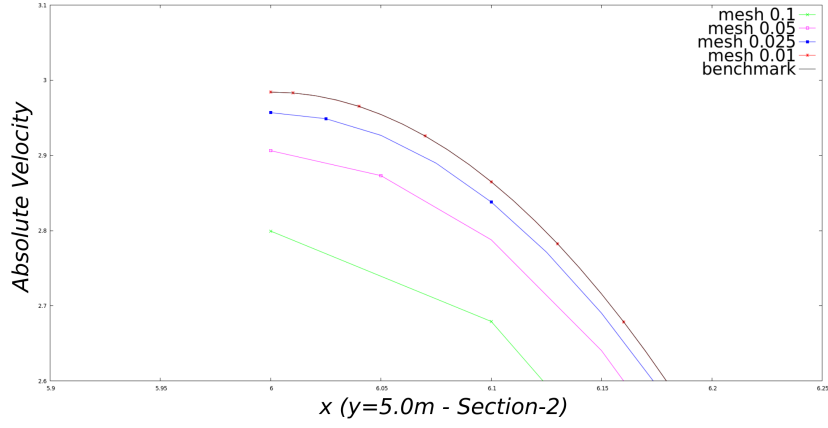

Fig. 17 Detailed view of the Fig. 15

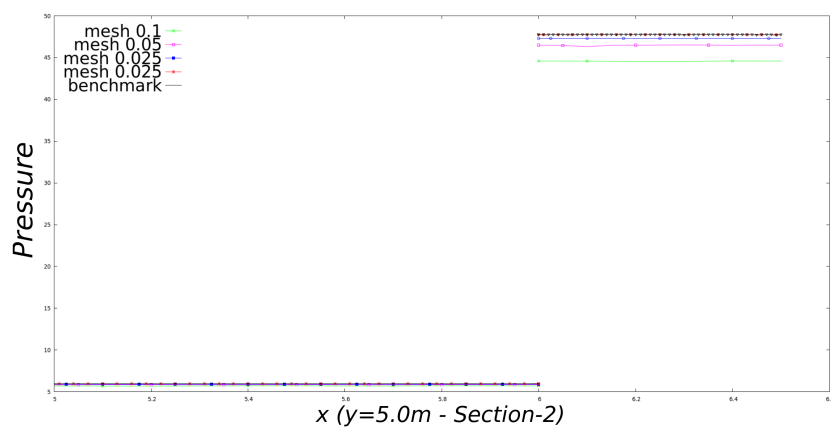

Fig. 18 Comparison of the pressures for different mesh settings and the benchmark solution on the Section-2

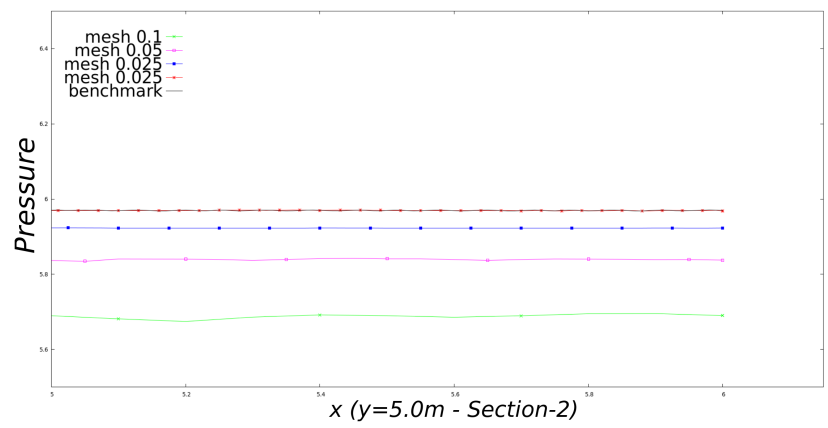

Fig. 19 Detailed view of the Fig. 18

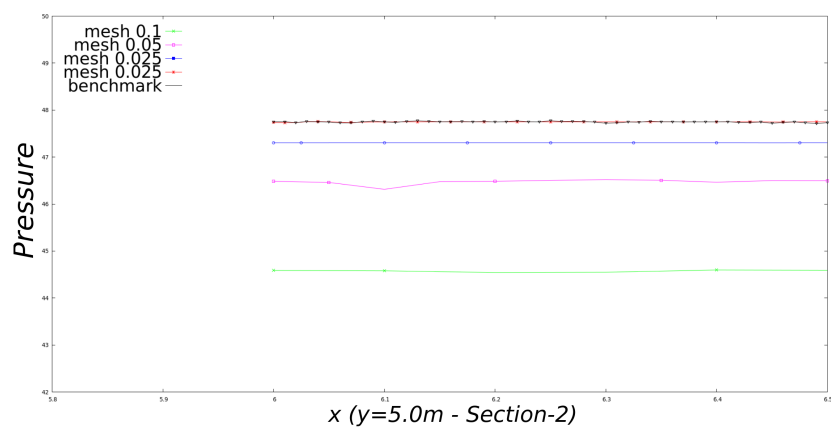

Fig. 20 Detailed view of the Fig. 18

According to [23], a rectangular domain of $1 \mathrm{~m}$ width and $4 m$ height has been used. The dense fluid on the top had a density of $\rho=1.225 \mathrm{~kg} / \mathrm{m}^{3}$, while the one 
on the bottom had $\rho=0.1694 \mathrm{~kg} / \mathrm{m}^{3}$. Both of the fluids had a dynamic-viscosity of $\mu=0.00313 \mathrm{~kg} /(\mathrm{ms})$. The effects of surface tension have been neglected, so that they were not considered in the simulation. The initial position of the interface had been taken as in the following function: $y(x)=2.0+0.05 \cos (2 \pi x)$. The simulation domain is provided on the Fig. 22 .

For the simulation, the mesh size has been chosen to be $h=0.025 \mathrm{~m}$ in an unstructured setting, while the time step was set as $d t=0.001 s$. Each element has been seeded with 45 particles initially, that had been distributed following the positions of corresponding gauss points inside the element. Simulation had been previously run with 6 and 15 particles on each element. Comparison of the results at $t=0.8$ for these three particle settings have been provided on the Fig. 21 as on the very left is the benchmark solution, 6 particles setting as the second, 15 particles as the as the third and the 45 particle setting as the last one on the right. Inspection of the results reveals that, although the results in the 6 and 15 particle settings are fairly good, the 45 particle setting provides better results. The current implementation of PFEM-2 uses a first order projection method for the transfer of the information from the particles onto the fixed mesh. Such choice is inaccurate and justifies the importance of using a large number of particles. This behaviour could be corrected by the use of a second order projection [39], which is however not explored in the current work.

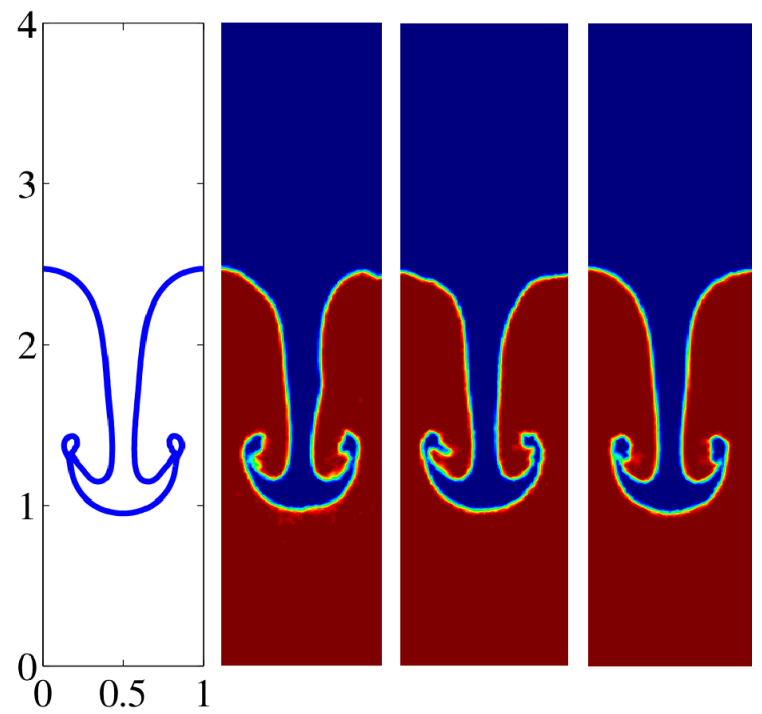

Fig. 21 Position of the interface at time $=0.8 \mathrm{~s}$ for the benchmark solution, 6, 15 and 45 particle settings, respectively

The position of the interface at the time $=0,0.7,0.8$ and $0.9 \mathrm{~s}$ have been compared with the results in [23] in the Fig. 23 and in Fig. 24. It should be noted that in [23] a mesh with triangular elements with a nonconstant characteristic element size as $h_{\text {min }}=0.01 \mathrm{~m}$ and $h_{\text {max }}=0.11 \mathrm{~m}$ had been used. Moreover in the further stages of the simulation, the mesh had been refined.

On the Fig. 23 and Fig. 24, the interfaces presented on the left are the results taken from the literature, while the ones on the right belong to the proposed methodology.

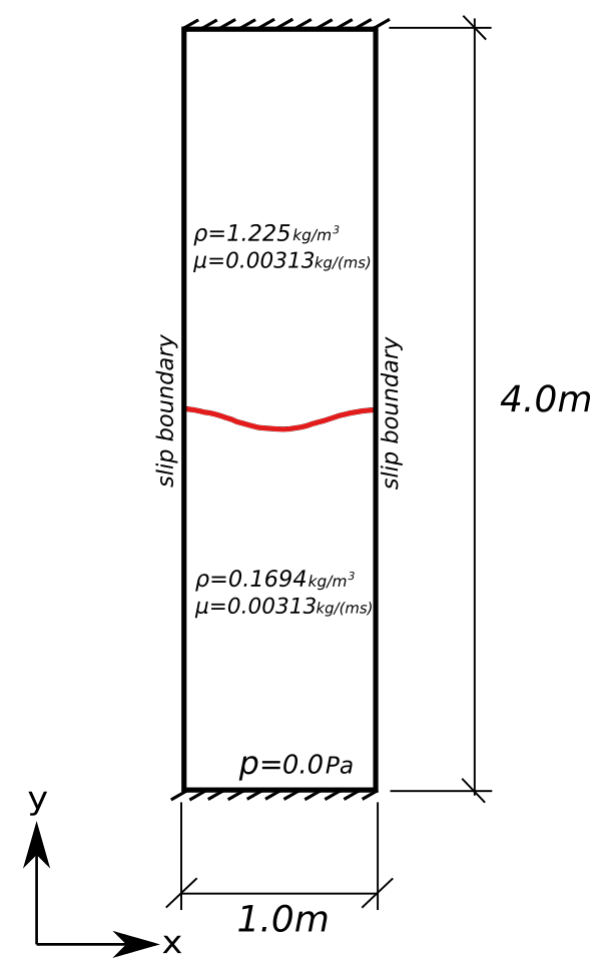

Fig. 22 Taylor-Rayleigh Instability domain

Inspection of the results reveal that, although a relatively coarse mesh has been employed, the methodology works fine in dynamic cases and provides similar results to the bibliography. However, based on the forming of the mushroom shape in the times 0.8 and 0.9 , it is has to be admitted that the Nitsche based methodology implemented in this work is not as successful as the ALE based approach presented in [23]. Nevertheless, considering the fact that the work in [23] had been employing a finer mesh from the very beginning, and an adaptivemesh refinement, it is fair to say that the methodology presented in this paper provides good results.

\subsection{Falling Drop Case}

In this test case, a material drop that falls inside a different surrounding material due to the gravitational 


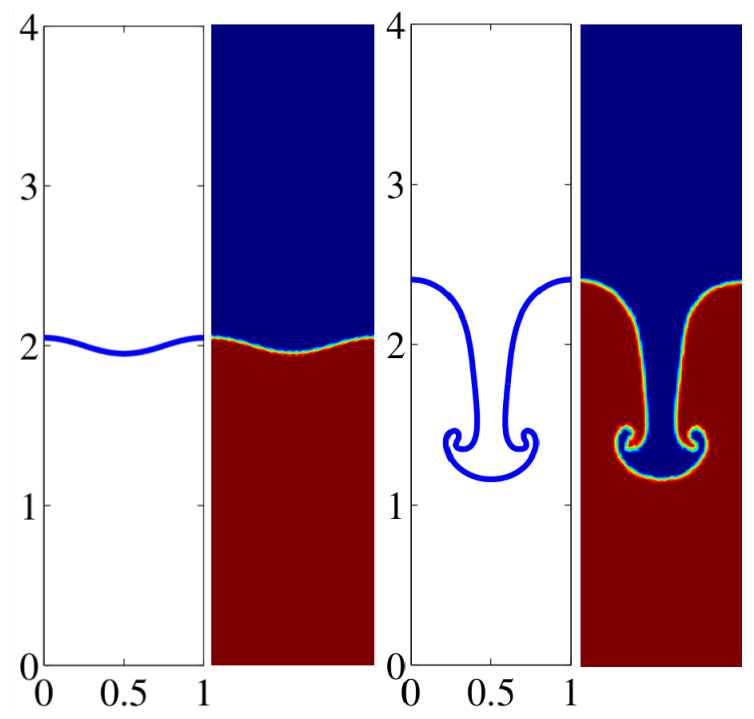

Fig. 23 Position of the interface at time $=0.0 \mathrm{~s}$ and $0.7 \mathrm{~s}-$ benchmark solution on the left vs. methodology on the right

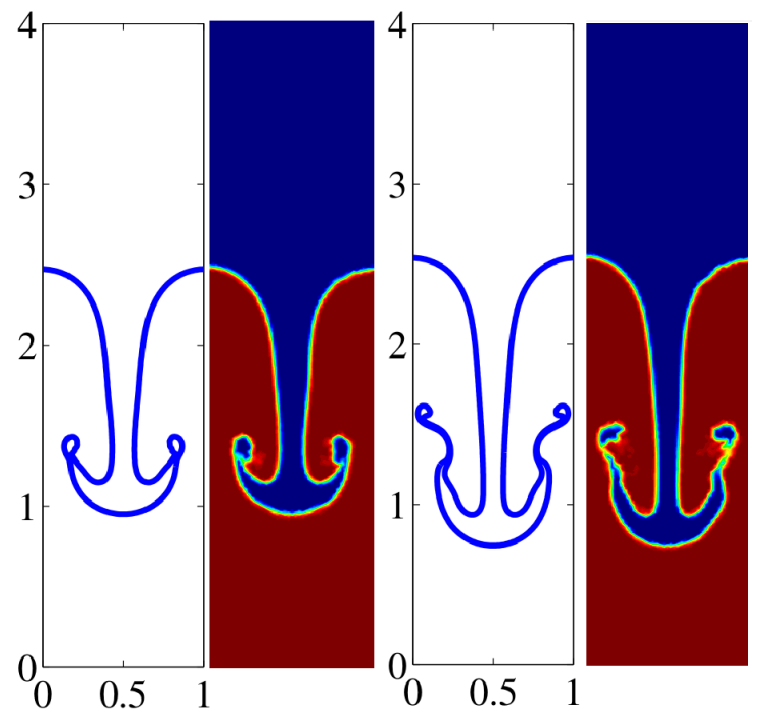

Fig. 24 Position of the interface at time $=0.8 \mathrm{~s}$ and $0.9 \mathrm{~s}-$ benchmark solution on the left vs. methodology on the right

forces has been simulated. For the initial setting, a circle function, $(x-0.5)^{2}+(y-1.5)^{2}=0.15^{2}$, to designate the locations of the drop and the surrounding material has been used. According to this, the area inside the circle defines the material region which the drop consists of, while the area out of the circle defines the material region which surrounds the falling drop. Both of the materials have been set to have unit values of density and dynamic viscosity, without body forces. The domain setting is provided on the Fig. 25.

A quick contemplation of this test case reveals that the physical phenomena here will never produce any kind of discontinuities in the flow field and there is no need to apply the Nitsche based methodology presented

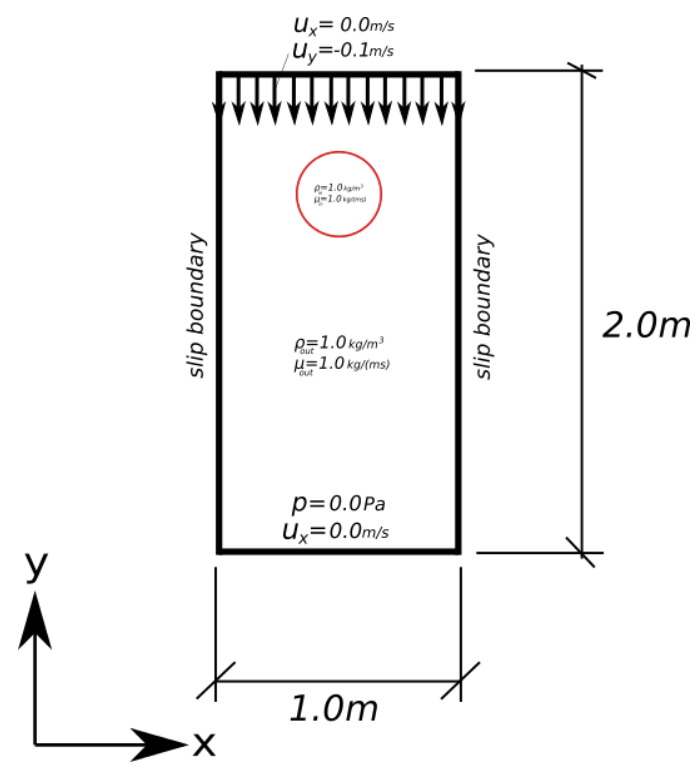

Fig. 25 Falling Drop domain

in this work. However, this test case has been set in this way to have a fair comparison of the methodology with classical FEM solution in terms of computational cost. To achieve this, the case has been simulated both with the Nitsche based methodology and the conventional FEM using a $h=0.025 \mathrm{~m}$ mesh setting on a computer hardware with the specifications of 8 cores Intel ${ }^{\circledR}$ $\mathrm{Core}^{\mathrm{TM}}$ i7-4790 CPU @ 3.60GHz with 8GB memory. After this, the time spent during different phases of the solution step have been compared. A direct solver that employs Skyline LU Factorization has been used. The comparison of the times has revealed that the total computational cost was $176.5494 \mathrm{~s}$ for the Nitsche based methodology, and it was 157.0246s for the nonenriched standard FEM solution. This shows that the methodology presented in this paper suffers from a $12 \%$ increment of computational cost. Apart from that, the Table. 1 presents the comparison of the specific time spent for each part of the solution procedure during the last time step.

\begin{tabular}{||c||cc||}
\hline \multicolumn{3}{||c||}{ Solution Times } \\
\hline Solution Part & Nitsche b. m. & Classical FEM \\
\hline \hline Setup Dof & 0.01970839 & 0.01411104 \\
\hline Setup System & $9.2983246 \mathrm{e}-05$ & $8.559227 \mathrm{e}-05$ \\
\hline Build & 0.0550902 & 0.029185 \\
\hline Solve & 0.683946 & 0.585278 \\
\hline
\end{tabular}

Table 1 Time measurement for the specific parts of the solution step all in seconds. 


\subsection{Sloshing}

As a last example, the so called "sloshing" test case, which had been previously studied in [19], has been tested. Furthermore, the results have been compared with the results in [19]. The results of the methodology used in [19] had been tested by comparing them with OpenFOAM software [27]. Therefore here, the results obtained with the new methodology will be compared both with the previous formulation, and OpenFOAM. The domain of the test case consists of two in-miscible fluids where the fluid on the top has a density of $\rho=1.0$ $\mathrm{kg} / \mathrm{m}^{3}$, while the one below has $\rho=1000.0 \mathrm{~kg} / \mathrm{m}^{3}$. Apart from that, both of the fluids have a dynamicviscosity of $\mu=0.000001 \mathrm{~kg} /(\mathrm{ms})$. Simulation domain has been selected to have a rectangular shape. Moreover, assuming the left corner of the domain to be the origin, the initial interface has been set to follow the function $y(x)=-0.25 x+0.4$. Apart from that, all the walls have been considered to follow "free-slip" boundary condition where the normal velocity on the wall has been set to zero and the flow in the tangential direction was free. The domain setting for the simulation is provided in the Fig. 26. The mesh used for this test case has been selected to be as $h=0.01 \mathrm{~m}$ and the time step size has been set to $d t=0.005 \mathrm{~s}$. It should be noted that in [19], the mesh size had been selected more than three times smaller, $h=0.003 \mathrm{~m}$. The comparisons of the interface for $\mathrm{t}=0.55 \mathrm{~s}, 1.7 \mathrm{~s}, 2.75 \mathrm{~s}, 3.35 \mathrm{~s}$ are presented in the Fig. 27.

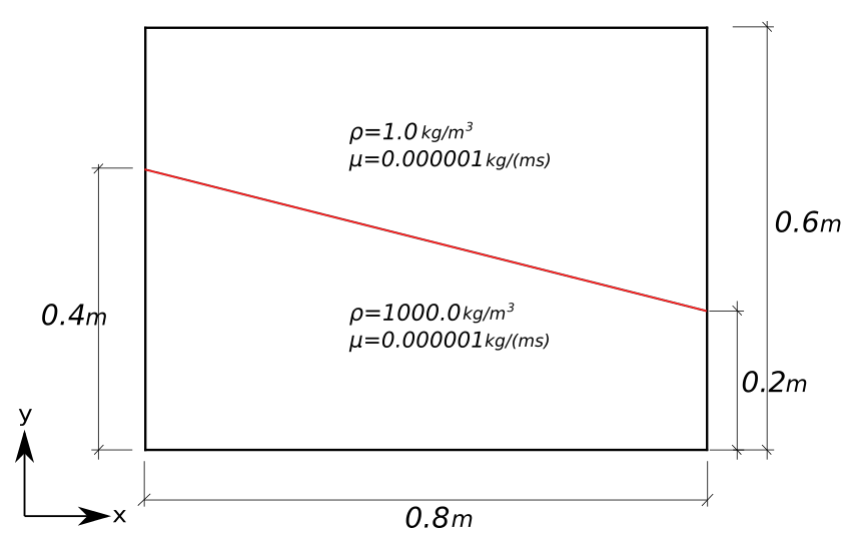

Fig. 26 Sloshing domain

Inspection of the results reveal that the new methodology, despite having a coarser mesh, is capable of capturing the same physics as in the other cases in the bibliography .

\section{Conclusion and Outlook}

In this work, a Nitsche based methodology has been combined with the PFEM-2 strategy to alleviate its discrepancies in capturing discontinuities in multi-fluid flow simulations. Three types of conditions where the boundary conditions are imposed weakly on the interface using Nitsche's method have been implemented. Later on, the methodology has been run on four test cases, where one of them was simulated with stationary Stokes Equations, while the other two with nonstationary Navier Stokes Equations with PFEM-2. The results have been compared either with benchmark solutions, or the bibliography. Despite using coarser mesh settings with respect to the benchmark or the bibliography, satisfying results with a reasonable compromise of the computational time have been achieved and it has been concluded that the method works properly and it is capable of capturing discontinuities.

The test cases have confirmed that the two conditions, the free slip condition and the continuous velocity condition, which are imposed weakly on the interface using the Nitsche based methodology, give accurate results. However, although it has been implemented, the third condition: continuous normal velocity condition has not been experimented in this work. This condition will definitely be studied, tested and published in the near future.

The methodology implemented in this work has been conducted only on $2 \mathrm{D}$, and its extension to $3 \mathrm{D}$ is expected to be straight-forward. In the upcoming steps of the research, the methodology will be implemented on $3 \mathrm{D}$, and necessary tests will be conducted.

Apart from the Nitsche based methodology employed in this paper, the PFEM-2 strategy used here was based on a first-order splitting method applied onto the Navier Stokes Equations. To improve the results of the overall methodology and the PFEM-2 strategy, a profound study will be conducted in the near future and a new variation of the PFEM-2 strategy that utilizes a secondorder splitting scheme will be implemented.

\section{Compliance with ethical standards}

Conflicts of interest: The authors declare that they have no conflict of interest.

Acknowledgements The research that has been presented in this publication and the results obtained have been conducted and achieved with the support of the Ministerio de Economía y Competitividad (MINECO) from Spain and its funding program Ayudas para Contratos Predoctorales para la Formación de Doctores (ref. BES-2014-070613). Author Deniz C. Tanyildiz would like to express special thanks to the 


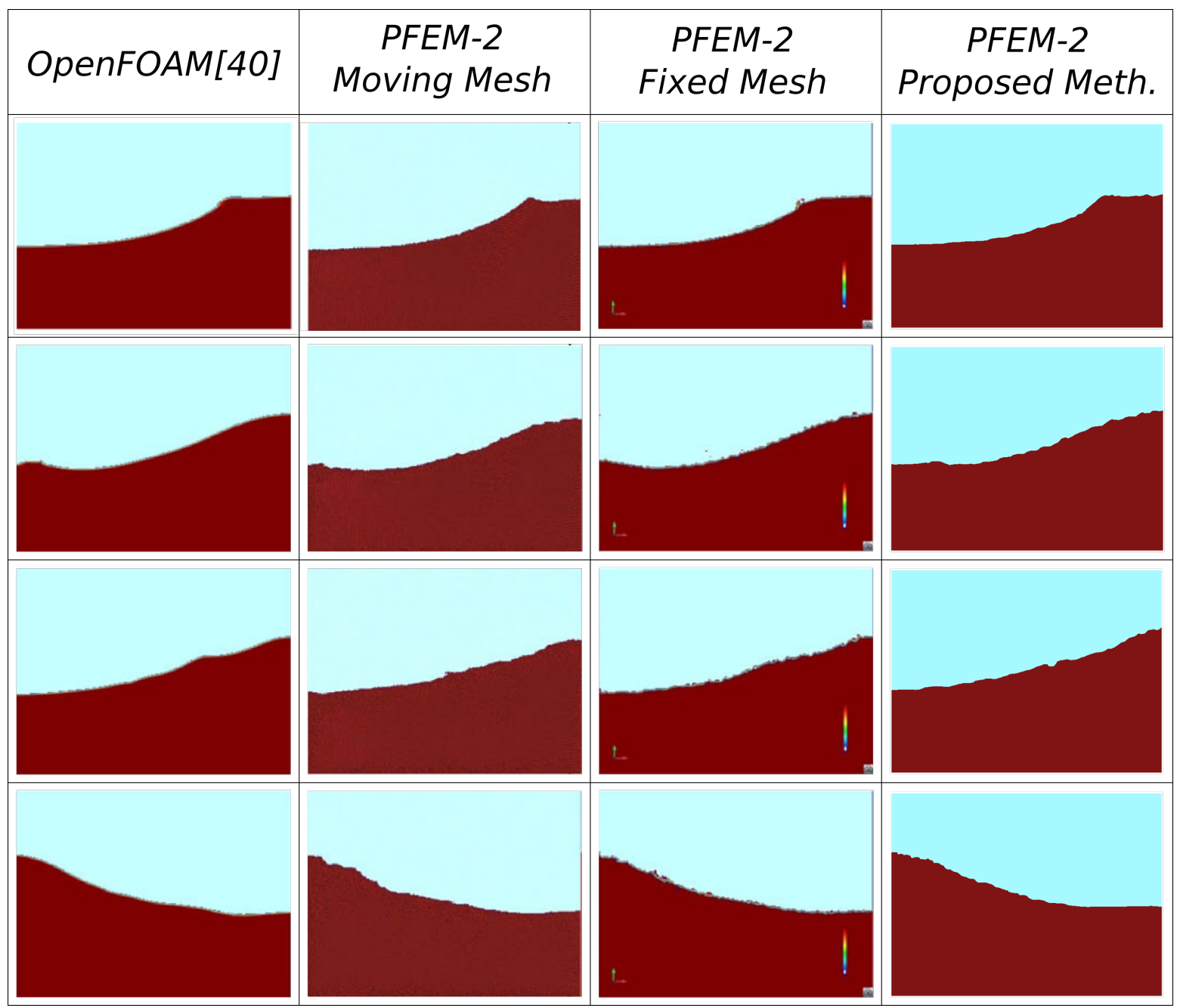

Fig. 27 Sloshing results comparison for $\mathrm{t}=0.55 \mathrm{~s}, 1.7 \mathrm{~s}, 2.75 \mathrm{~s}, 3.35 \mathrm{~s}$ at each row

project: PARFLOW(ref. BIA2013-49007-C2-1-R). Dr. Riccardo Rossi would like to express special thanks to the project: EXAQUTE(ref. 800898). Moreover, the authors would like to express their gratitude to Dr. Joan Baiges from Polytechnical University of Catalonia, for his substantial help on Nitsche's Method.

\section{References}

1. Osher SJ, Fedkiw RP. Level Set Methods and Dynamic Implicit Surfaces. Springer. New York. 2006.

2. Rossi R, Larese A, Dadvand P, Oñate E. An efficient edgebased level set finite element method for free surface flow problems. International Journal for Numerical Methods in Fluids. 2013. 71(6):687-716.

3. Cyril HW, Nichols BD. Volume of fluid (VOF) method for the dynamics of free boundaries. Journal of Computational Physics. 1981. 39(1):201-225.

4. Ausas RF, Dari EA, Buscaglia GC. A geometric masspreservating redistancing scheme for the level set function. International Journal of Numerical Methods in Fluids 2011. 65(8):989-1010.

5. Coppola-Owen AH, Codina R. Improving Eulerian twophase flow finite element approximation with discontinuous gradient pressure shape functions. International Journal for Numerical Methods in Fluids. 2005. 49(12):1287-1304.

6. Cruchaga M, Celentano D, Tezduyar T. A moving Lagrangian interface technique for flow computations over fixed meshes. Computer Methods in Applied Mechanics and Engineering 2001. 191(6):525-543.

7. Barton PT, Obadia B, Drikakis D. A conservative level-set method for compressible solid/fluid problems on fixed grids. Journal of Computational Physics. 2011. 230:7867-7890.

8. Spelt P DM. A level-set approach for simulations of flows with multiple moving contact lines with hysteresis. Journal of Computational Physics 2005. 207(2). 389-404.

9. Zhang YL, Zou QP, Greaves D. Numerical simulation of free-surface flow using the level-set method with global mass correction. International Journal of Numerical Methods in Fluids 2010. 63(6):366-396.

10. Idelsohn S, de Mier-Torrecilla M, Oñate E. Multi-fluid flows with the particle finite element method. Computer Methods in Applied Mechanics and Engineering 2009. 198(33).2750-2767.

11. Mier-Torrecilla M. Numerical simulation of multi-fluid flows with the particle finite element method. Ph.D. Thesis. 2010 .

12. Idelsohn SR, Mier-Torrecilla M, Marti J, Oñate E. Editor:Oñate, E and Owen, R. The Particle Finite Element 
Method for Multi-Fluid Flows. Particle-Based Methods: Fundamentals and Applications. 2011. Springer Netherlands. Dordrecht. 135-158.

13. Marti J, Ryzhakov P B, Idelsohn SR, Oñate E. Combined Eulerian-PFEM approach for analysis of polymers in fire situations. International Journal for Numerical Methods in Engineering. 2012. 92(9). 782-801.

14. Ryzhakov P B, Jarauta A. An embedded approach for immiscible multi-fluid problems. Int. J. Numer. Meth. Fluids. 2016. 81:357-376.

15. Jarauta A, Ryzhakov P B, Secanell M, Waghmare P, Pons-Prats J. Numerical study of droplet dynamics in a polymer electrolyte fuel cell gas channel using an embedded Eulerian-Lagrangian approach. Journal of Power Sources. 2016. 323. 201-212.

16. Bathe K.J. The inf-sup condition and its evaluation for mixed finite element methods. Comput. Struct. 2001. 79. 243-252.

17. Fries TP, The intrinsic XFEM for two-fluid flows, Int. J. Numer. Meth. Fluids. 2009. 60:437-471.

18. Idelsohn SR, Nigro N, Limache A, Oñate E. Large timestep explicit integration method for solving problems with dominant convection. Computer Methods in Applied Mechanics and Engineering. 2012. 217-220. 168-185.

19. Idelsohn SR, Marti J, Becker PA, Oñate E. Analysis of multifluid flows with large time steps using the particle finite element method. International Journal for Numerical Methods in Fluids. 2014. 75. 621-644.

20. Becker PA, Idelsohn SR, Oñate E. A unified monolithic approach for multi-fluid flows and fluid-structure interaction using the Particle Finite Element Method with fixed mesh. Computational Mechanics. 2015. 55. 1091-1104.

21. Hansbo P, Hansbo A. An unfitted finite element method, based on Nitsche's method, for elliptic interface problems. Computer Methods in Applied Mechanics and Engineering. 2002. 191(47-48). 5537-5552.

22. Nitsche J. Über ein Variationsprinzip zur Lösung von Dirichlet-Problemen bei Verwendung von Teilräumen, die keinen Randbedingungen unterworfen sind. Abhandlungen aus dem Mathematischen Seminar der Universität Hamburg. 1971. 36. 9-15.

23. Ganesan S, Tobiska L, Computations on flows with interfaces using arbitrary Langrangian Eulerian method. European Conference on Computational Fluid Synamics. 2006. ECCOMAS CFD.

24. Idelsohn SR, Gimenez JM, Nigro NM. Multifluid flows with weak and strong discontinuous interfaces using an elemental enriched space. Int J Numer Meth Fluids. 2018. 86:750-769.

25. Idelsohn SR, Gimenez J, Marti J and Nigro N. Elemental enriched spaces for the treatment of weak and strong discontinuous fields. Computer methods in applied mechanics and engineering. 2017. 313: 535-559.

26. Marti J, Ortega E and Idelsohn SR. An improved enrichment method for weak discontinuities for thermal problems. International journal of numerical methods for heat \& fluid flow. 2017. 27(8). 1748-764.

27. Hrvoje J, Jemcov A, Tukovic Z. OPEN FOAM: A C++ library for complex physics simulations. Int. Workshop Coupled Methods Numer. Dyn. IUC, Dubrovnik, Croatia, 2007.

28. Marti J., Idelsohn S.R. and Oñate E, A Finite Element Model for the Simulation of the UL-94 Burning Test, Fire Technology, Fire Technology. 2018. 54(6). 1783-1805, .

29. Ryzhakov PB, Marti J., Idelsohn SR and Oñate E, Fast fluid-structure interaction simulations using a displacement-based finite element model equipped with an explicit streamline integration prediction, Computer methods in applied mechanics and engineering. 2017. 315. 10801097.

30. Ryzhakov PB, Cotela J, Rossi R, and Oñate E. A twostep monolithic method for the efficient simulation of incompressible flows. International Journal for Numerical Methods in Fluids. 2014. 74(12). 919-934.

31. Hughes TJR. Multiscale phenomena: Green's functions, the Dirichlet-to-Neumann formulation, subgrid scale models, bubbles and the origins of stabilized. Computer Methods in Applied Mechanics and Engineering. 1995. 73. 387401.

32. Dadvand P, Rossi R, Oñate E. An object-oriented environment for developing finite element codes for multidisciplinary applications. Archives of Computational Methods in Engineering. 2010. 17/3. 253-297.

33. Dadvand P, Rossi R, Gil M, Martorell X, Cotela J, Juanpere E, Idelsohn SR, Oñate E. Migration of a generic multiphysics framework to HPC environments. Computers \& Fluids. 2013. 80. 301-309.

34. Oñate E, and Idelsohn SR, Del Pin F and Romain A. The particle finite element method: an overview. International Journal of Computational Methods. 2004. 1(02). 267-307.

35. Cremonesi M, Ferri F and Perego U. A basal slip model for Lagrangian finite element simulations of 3D landslides. International Journal for Numerical and Analytical Methods in Geomechanics. 2017. 41. 30-53.

36. Marti J, Ryzhakov PB. An explicit-implicit finite element model for the numerical solution of incompressible Navier-Stokes equations on moving grids. Computer Methods in Applied Mechanics and Engineering. 2019. 350. 750765 .

37. Idelsohn SR, Nigro N, Gimenez JM, Rossi R, Marti J. A fast and accurate method to solve the incompressible Navier-Stokes equations. Engineering Computations. 2013. 30. $197-222$

38. Becker P. An enhanced Particle Finite Element Method with special emphasis on landslides and debris flows. PhD Thesis. Universitat Politècnica de Catalunya 2015.

39. Gimenez JM, Horacio JA, Idelsohn SR, Nigro NM. A second-order in time and space particle-based method to solve flow problems on arbitrary meshes. Journal of Computational Physics. 2019. 380. 295-310. 